\title{
On an extension of contractivity conditions via auxiliary functions
}

Ravi P Agarwal ${ }^{1,2,3}$, Erdal Karapınar ${ }^{3,4^{*}}$ and Antonio-Francisco Roldán-López-de-Hierro ${ }^{5}$

\section{"Correspondence:}

erdal.karapinar@atilim.edu.tr

${ }^{3}$ Nonlinear Analysis and Applied

Mathematics Research Group

(NAAM), King Abdulaziz University,

Jeddah, Saudi Arabia

${ }^{4}$ Department of Mathematics,

Atilim University, Incek, Ankara,

06836, Turkey

Full list of author information is

available at the end of the article

\begin{abstract}
In this manuscript, we study sufficient conditions on the functions that appears in very complex contractivity conditions introduced in a recent manuscript by Liu et al. in order to guarantee the existence and uniqueness of common fixed points of four self-mappings.
\end{abstract}

Keywords: fixed point theorem; fixed point; common fixed point; contractivity condition

\section{Introduction}

Originally, a Banach contractive mapping [1] is a mapping $T: X \rightarrow X$ from a metric space $(X, d)$ into itself for which there exists $\lambda \in[0,1)$ such that

$$
d(T x, T y) \leq \lambda d(x, y) \quad \text { for all } x, y \in X .
$$

In [2], Rhoades did a complete study about different notions of contractive mapping that were appearing after Banach's pioneering notion, including, among others, the following ones:

- Kannan [3], 1969,

$$
d(T x, T y) \leq \lambda(d(x, T x)+d(y, T y)), \quad \text { where } \lambda \in[0,1 / 2) .
$$

- Reich [4], 1971,

$$
d(T x, T y) \leq a d(x, T x)+b d(y, T y)+c d(x, y), \quad \text { where } a+b+c<1 .
$$

- Bianchini [5], 1972,

$$
d(T x, T y) \leq \lambda \max \{d(x, T x), d(y, T y)\}, \quad \text { where } \lambda \in[0,1) .
$$

- Chatterjea [6], 1972,

$$
d(T x, T y) \leq \lambda(d(x, T y)+d(y, T x)), \quad \text { where } \lambda \in[0,1 / 2) .
$$

\section{Springer}


- Sehgal [7], 1972,

$$
d(T x, T y)<\max \{d(x, T x), d(y, T y), d(x, y)\} .
$$

- Hardy and Rogers [8], 1973,

$$
\begin{aligned}
& d(T x, T y) \leq a_{1} d(x, y)+a_{2} d(x, T x)+a_{3} d(y, T y)+a_{4} d(x, T y)+a_{5} d(y, T x), \\
& \text { where } a_{1}+a_{2}+a_{3}+a_{1}+a_{5}<1 .
\end{aligned}
$$

- Ćirić [9], 1974,

$$
\begin{aligned}
& d(T x, T y) \leq \lambda \max \{d(x, y), d(x, T x), d(y, T y), d(x, T y), d(y, T x)\}, \\
& \text { where } \lambda \in[0,1) .
\end{aligned}
$$

Later, some auxiliary functions were appearing in the contractivity condition. To cite some of them, the following ones are well-known contractivity conditions.

- Boyd and Wong [10], 1969,

$$
d(T x, T y) \leq \varphi(d(x, y)) .
$$

- Rhoades [11], 2001,

$$
d(T x, T y) \leq d(x, y)-\phi(d(x, y))
$$

- Dutta and Choudhury [12], 2008,

$$
\psi(d(T x, T y)) \leq \psi(d(x, y))-\phi(d(x, y)) .
$$

- Aydi et al. [13], 2013,

$$
\psi(d(T x, T y)) \leq \psi(M(x, y))-\phi(M(x, y))+L N(x, y)
$$

where $L \geq 0$,

$$
\begin{aligned}
& M(x, y)=\max \left\{d(x, y), d(x, T x), d(y, T y), \frac{d(x, T y)+d(y, T x)}{2}\right\} \text { and } \\
& N(x, y)=\max \{d(x, T x), d(y, T y), d(x, T y), d(y, T x)\} .
\end{aligned}
$$

- Berzig et al. [14], 2014,

$$
\psi(d(T x, T y)) \leq \alpha(x, y) \psi(M(x, y))-\beta(x, y) \varphi(M(x, y)),
$$

where $M(x, y)$ is given by one of the following cases:

$$
\text { - } M_{1}(x, y)=\max \left\{d(x, y), d(x, T x), d(y, T y), \frac{d(x, T y)+d(y, T x)}{2}\right\} \quad \text { (type I); }
$$


- $\quad M_{2}(x, y)=\max \{d(x, y), d(x, T x), d(y, T y)\} \quad$ (type II);

- $M_{3}(x, y)=\max \left\{d(x, y), \frac{d(x, T x)+d(y, T y)}{2}, \frac{d(x, T y)+d(y, T x)}{2}\right\} \quad$ (type III);

- $M_{4}(x, y)=\max \left\{d(x, y), \frac{d(x, T x)+d(y, T y)}{2}\right\} \quad$ (type IV);

- $M_{5}(x, y)=d(x, y) \quad($ type V).

After the appearance of Rhoades' theorem (see [11]), many results have been introduced involving contractivity conditions in which some families of functions play a key role, even in partially ordered metric spaces (see, for instance, [12, 14-33]). As we can observe, each time, more and more terms have been included in the right-hand side of the contractivity inequality. As a consequence, new results are getting better.

In a very recent manuscript, Liu et al. [34] introduced common fixed point theorems for self-mappings $A, B, T, S: X \rightarrow X$ involving some very complex contractivity conditions, as the following one.

Theorem 1 (Liu et al. [34], Theorem 2.1) Let A, B, S, and T be self-mappings in a metric space $(X, d)$ such that

$\{A, T\}$ and $\{B, S\}$ are weakly compatible;

$T(X) \subseteq B(X)$ and $S(X) \subseteq A(X) ;$

one of $A(X), B(X), S(X)$, and $T(X)$ is complete;

$d(T x, S y) \leq \psi\left(M_{1}(x, y)\right), \quad \forall x, y \in X$,

where $\psi_{1}$ is in $\Phi_{3}$ and $M_{1}$ is defined, for all $x, y \in X$, by

$$
\begin{aligned}
M_{1}(x, y)= & \max \left\{d(A x, B y), d(A x, T x), d(B y, S y), \frac{d(A x, S y)+d(T x, B y)}{2},\right. \\
& \frac{d(A x, S y) d(T x, B y)}{1+d(A x, B y)}, \frac{d(A x, T x) d(B y, S y)}{1+d(A x, B y)}, \\
& \left.\frac{1+d(A x, S y)+d(T x, B y)}{1+d(A x, T x)+d(B y, S y)} d(A x, T x)\right\} .
\end{aligned}
$$

Then $A, B$, S, and T have a unique common fixed point in $X$.

Other statements were proved in the mentioned paper including similar contractivity conditions involving functions $M_{2}, M_{3}: X^{2} \rightarrow[0, \infty)$ as follows:

$$
\begin{aligned}
M_{2}(x, y)= & \max \left\{d(A x, B y), d(A x, T x), d(B y, S y), \frac{d(A x, S y)+d(T x, B y)}{2},\right. \\
& \frac{1+d(A x, T x)}{1+d(A x, B y)} d(B y, S y), \frac{1+d(B y, S y)}{1+d(A x, B y)} d(A x, T x), \\
& \left.\frac{1+d(A x, S y)+d(T x, B y)}{1+d(A x, T x)+d(B y, S y)} d(B y, S y)\right\} \text { and }
\end{aligned}
$$




$$
\begin{aligned}
M_{3}(x, y)= & \max \left\{d(A x, B y), d(A x, T x), d(B y, S y), \frac{d(A x, S y)+d(T x, B y)}{2},\right. \\
& \left.\frac{d(A x, S y) d(T x, B y)}{1+d(A x, B y)}\right\} .
\end{aligned}
$$

Obviously, in the future, new contractivity conditions will appear involving more and more terms in the right-hand side of the contractivity inequality. It is not our purpose to complicate such situation. On the contrary, the main aim of the present manuscript is to study what kind of functions might we include in an efficient contractivity condition depending on the terms we wish to consider (like $d(A x, B y), d(A x, T x), d(B y, S y)$, etc.). To do that, we present some families of very general functions that we can use in a contractivity condition so that Theorem 1 remains true. In other words, for instance, we illustrate what kind of functions we could consider involving the terms $d(A x, S y)$ and $d(T x, B y)$. Thus, we show how we can replace $(d(A x, S y)+d(T x, B y)) / 2$ in (2) by another term $\phi_{1}(d(A x, S y), d(T x, B y))$ considering a function $\phi_{1}$, which generalizes the particular case $\phi(t, s)=(t+s) / 2$. It is our purpose that, in the future, when some authors wish to include new terms in the contractivity conditions, they first ponder on what the sufficient conditions are on the functions involved in the arguments of the contractivity condition in order for their main statements to remain true.

\section{Preliminaries}

We will follow notations given in [34]. Throughout this paper, $\mathbb{N}$ denotes the set of all positive integers, $\mathbb{N}_{0}=\mathbb{N} \cup\{0\}$, and $X$ stands for a nonempty set. Given $n \in \mathbb{N}, X^{n}$ will denote the Cartesian space $X \times X \times \stackrel{(n)}{\cdots} \times X$ of $n$ identical copies of $X$.

Definition 2 A fixed point of a self-mapping $T: X \rightarrow X$ is a point $x \in X$ such that $T x=x$. A coincidence point of two or more operators $T_{1}, T_{2}, \ldots, T_{n}: X \rightarrow X$ is a point $x \in X$ such that $T_{1} x=T_{2} x=\cdots=T_{n} x$. A common fixed point of two or more operators $T_{1}, T_{2}, \ldots, T_{n}$ : $X \rightarrow X$ is a point $x \in X$ such that $T_{1} x=T_{2} x=\cdots=T_{n} x=x$.

Definition 3 Given a nonempty set $X$, a pair $\{T, g\}$ of self-mappings $T, g: X \rightarrow X$ are said to be weakly compatible if $\operatorname{Tg} x=g T x$ for all $x \in X$ such that $T x=g x$.

Let $\Phi_{1}, \Phi_{2}$, and $\Phi_{3}$ the following families of control functions:

$$
\begin{aligned}
\Phi_{1}= & \left\{\phi:[0, \infty) \rightarrow[0, \infty): \phi \text { is continuous, nondecreasing and } \phi^{-1}(\{0\})=\{0\}\right\}, \\
\Phi_{2}= & \left\{\phi:[0, \infty) \rightarrow[0, \infty): \phi \text { is lower semi-continuous and } \phi^{-1}(\{0\})=\{0\}\right\}, \text { and } \\
\Phi_{3}= & \{\phi:[0, \infty) \rightarrow[0, \infty): \phi \text { is upper semi-continuous, and } \\
& \left.\lim _{n \rightarrow \infty} a_{n}=0 \text { for each sequence }\left\{a_{n}\right\}_{n \in \mathbb{N}} \subset[0, \infty) \text { with } a_{n+1} \leq \phi\left(a_{n}\right), \forall n \in \mathbb{N}\right\} .
\end{aligned}
$$

The functions in $\Phi_{1}$ are known as altering distance functions (see [35]).

Lemma 4 ([34], Lemma 1.1) If $\phi \in \Phi_{3}$, then $\phi(0)=0$ and $\phi(t)<t$ for all $t>0$.

Henceforth, $(X, d)$ stands for a metric space. 
Proposition 5 Let $(X, d)$ be a metric space and let $\left\{x_{n}\right\} \subseteq X$ be a sequence such that $\lim _{n \rightarrow \infty} d\left(x_{n}, x_{n+1}\right)=0$. If $\left\{x_{n}\right\}$ is not a Cauchy sequence, then $\left\{x_{2 n}\right\}$ is not a Cauchy sequence. In particular, there exist $\varepsilon_{0}>0$ and two subsequences $\left\{x_{2 n(k)}\right\}$ and $\left\{x_{2 m(k)}\right\}$ of $\left\{x_{2 n}\right\}$ such that

$$
\begin{aligned}
2 k<2 n(k)<2 m(k), & d\left(x_{2 n(k)}, x_{2 m(k)-2}\right) \leq \varepsilon_{0}<d\left(x_{2 n(k)}, x_{2 m(k)}\right) \quad \text { for all } k \in \mathbb{N}, \\
\lim _{n \rightarrow \infty} d\left(x_{2 n(k)}, x_{2 m(k)}\right)= & \lim _{n \rightarrow \infty} d\left(x_{2 n(k)}, x_{2 m(k)-1}\right)=\lim _{n \rightarrow \infty} d\left(x_{2 n(k)}, x_{2 m(k)+1}\right) \\
= & \lim _{n \rightarrow \infty} d\left(x_{2 n(k)-1}, x_{2 m(k)-1}\right)=\lim _{n \rightarrow \infty} d\left(x_{2 n(k)-1}, x_{2 m(k)+1}\right)=\varepsilon_{0} .
\end{aligned}
$$

Proof By contradiction, assume that $\left\{x_{2 n}\right\}$ is a Cauchy sequence. Let $\varepsilon>0$ be arbitrary. Since $\left\{d\left(x_{n}, x_{n+1}\right)\right\} \rightarrow 0$, there exists $n_{1} \in \mathbb{N}$ such that

$$
d\left(x_{n}, x_{n+1}\right) \leq \frac{\varepsilon}{3} \text { for all } n \geq n_{1}
$$

As $\left\{x_{2 n}\right\}$ is a Cauchy sequence, there exists $n_{2} \in \mathbb{N}$ such that

$$
d\left(x_{2 n}, x_{2 m}\right) \leq \frac{\varepsilon}{3} \quad \text { for all } m, n \in \mathbb{N} \text { such that } 2 m \geq 2 n \geq n_{2} .
$$

Let $n_{0}=\max \left\{n_{1}, n_{2}\right\}$ and let $n, m \in \mathbb{N}$ be such that $m>n>n_{0}$. Let define

$$
p=\left\{\begin{array}{ll}
n / 2, & \text { if } n \text { is even, } \\
(n+1) / 2, & \text { if } n \text { is odd; }
\end{array} \quad q= \begin{cases}m / 2, & \text { if } m \text { is even } \\
(m+1) / 2, & \text { if } m \text { is odd }\end{cases}\right.
$$

Then $2 p=n$ if $n$ is even and $2 p=n+1$ if $n$ is odd. Similarly, $2 q=m$ if $m$ is even and $2 q=m+1$ if $m$ is odd. Since $2 q \geq 2 p \geq n_{2}$,

$$
d\left(x_{n}, x_{m}\right) \leq d\left(x_{n}, x_{2 p}\right)+d\left(x_{2 p}, x_{2 q}\right)+d\left(x_{2 q}, x_{m}\right) \leq \frac{\varepsilon}{3}+\frac{\varepsilon}{3}+\frac{\varepsilon}{3}=\varepsilon .
$$

This means that $\left\{x_{n}\right\}$ is a Cauchy sequence, which is false. As a consequence, $\left\{x_{2 n}\right\}$ cannot be a Cauchy sequence.

\section{Common fixed point theorems in metric spaces}

For convenience, we introduce the following families of functions. Let $F_{1}$ be the family of all functions $\phi:[0, \infty)^{n} \rightarrow[0, \infty)$, with $n \geq 2$, such that, for all $r, z_{3}, z_{4}, \ldots, z_{n} \in[0, \infty)$ :

$\left(\mathrm{F}_{11}\right) \phi$ is continuous on its first two arguments;

$\left(\mathrm{F}_{12}\right) \phi\left(0, r, z_{3}, z_{4}, \ldots, z_{n}\right) \leq \frac{r}{2}$;

$\left(\mathrm{F}_{13}\right) \phi\left(r, r, z_{3}, z_{4}, \ldots, z_{n}\right) \leq r$ for all $r>0$;

$\left(\mathrm{F}_{14}\right) \phi\left(r, 0, z_{3}, z_{4}, \ldots, z_{n}\right) \leq r$ for all $r>0$.

Examples of functions in $F_{1}$ are the following ones:

$$
\begin{aligned}
& \phi(r, s)=\frac{r+s}{2} ; \\
& \phi(r, s)=\max \left\{r, \frac{s}{2}\right\} ;
\end{aligned}
$$




$$
\begin{aligned}
& \phi(r, s)=\max \left\{r, \frac{r+s}{2}\right\} ; \\
& \phi(r, s, t)=\frac{1}{1+t^{2}} \frac{r+s}{2} ; \\
& \phi(r, s, t, u)=\frac{\mathrm{e}^{-u^{2}}}{1+t^{4}} \max \left\{r, \frac{r+s}{2}\right\} .
\end{aligned}
$$

Let $\mathrm{F}_{2}$ be the family of all functions $\phi:[0, \infty)^{n} \rightarrow[0, \infty)$, with $n \geq 3$, such that, for all $r, s, z_{4}, z_{5}, \ldots, z_{n} \in[0, \infty)$ :

$\left(\mathrm{F}_{21}\right) \phi$ is continuous on its first three arguments;

$\left(\mathrm{F}_{22}\right) \phi\left(0, r, s, z_{4}, z_{5}, \ldots, z_{n}\right) \leq \max \left\{\frac{r}{2}, s\right\}$;

$\left(\mathrm{F}_{23}\right) \phi\left(r, r, r, z_{4}, z_{5}, \ldots, z_{n}\right) \leq r$ for all $r>0$;

$\left(\mathrm{F}_{24}\right) \phi\left(r, 0,0, z_{4}, z_{5}, \ldots, z_{n}\right) \leq r$ for all $r>0$.

Examples of functions in $\mathrm{F}_{2}$ are the following ones:

$$
\begin{aligned}
& \phi(r, s, t)=\frac{r s}{L+t} \quad \text { where } L>0 \\
& \phi(r, s, t)=\frac{r s}{L+t+\mathrm{e}^{t}} \quad \text { where } L>0 \\
& \phi\left(r, t, s, z_{1}, z_{2}\right)=\max \{r, s, t\} .
\end{aligned}
$$

Let $F_{3}$ be the family of all functions $\phi:[0, \infty)^{n} \rightarrow[0, \infty)$, with $n \geq 3$, such that, for all $r, s, z_{4}, z_{5}, \ldots, z_{n} \in[0, \infty)$ :

$\left(\mathrm{F}_{31}\right) \phi$ is continuous on its first three arguments;

$\left(\mathrm{F}_{32}\right) \phi\left(r, s, s, z_{4}, z_{5}, \ldots, z_{n}\right) \leq \max \{r, s\}$

$\left(\mathrm{F}_{33}\right) \phi\left(0,0, r, z_{4}, z_{5}, \ldots, z_{n}\right) \leq r$ for all $r>0$;

$\left(\mathrm{F}_{34}\right) \phi\left(0, r, 0, z_{4}, z_{5}, \ldots, z_{n}\right) \leq r$ for all $r>0$.

For instance,

$$
\begin{aligned}
& \phi(r, s, t)=\frac{r s}{L+t} \quad \text { where } L>0, \\
& \phi\left(r, t, s, z_{1}, z_{2}\right)=\max \{r, s, t\} .
\end{aligned}
$$

Let $\mathrm{F}_{4}$ be the family of all functions $\phi:[0, \infty)^{n} \rightarrow[0, \infty)$, with $n \geq 4$, such that, for all $r, s, t, u, z_{3}, z_{4}, \ldots, z_{n} \in[0, \infty)$ :

$\left(\mathrm{F}_{41}\right) \phi$ is continuous on its first four arguments;

$\left(\mathrm{F}_{42}\right) \phi\left(0, r, s, t, z_{5}, z_{6}, \ldots, z_{n}\right) \leq \max \left\{\frac{r}{2}, s, t\right\}$;

$\left(\mathrm{F}_{43}\right) \phi\left(r, r, 0,0, z_{5}, z_{6}, \ldots, z_{n}\right) \leq r$ for all $r>0$;

$\left(\mathrm{F}_{44}\right) \phi\left(r, 0,0, r, z_{5}, z_{6}, \ldots, z_{n}\right) \leq r$ for all $r>0$.

For instance,

$$
\begin{aligned}
& \phi(r, s, t, u)=\frac{L+r+s}{L+t+u} t \quad \text { where } L>0 ; \\
& \phi(r, s, t, u)=\max \left\{\frac{r+s}{2}, t, u\right\} ;
\end{aligned}
$$




$$
\phi\left(r, s, t, u, z_{1}, z_{2}\right)=\frac{\arctan \left(z_{1}\right)}{2+z_{2}^{2}} \max \left\{\frac{r+s}{2}, t, u\right\}
$$

The main theorem of the present manuscript is the following one. Notice that we assume that $\phi_{1}$ has two arguments, $\phi_{2}$ and $\phi_{3}$ have three arguments and $\phi_{4}$ has four arguments because other arguments are not important through the proof.

Theorem 6 Let $A, B, S, T: X \rightarrow X$ be self-mappings in a metric space $(X, d)$ such that
(a) $\{A, T\}$ and $\{B, S\}$ are weakly compatible;
(b) $T(X) \subseteq B(X)$ and $S(X) \subseteq A(X)$;
(c) one of $A(X), B(X), S(X)$, and $T(X)$ is complete.

Also assume that there exist $\phi_{1} \in \mathrm{F}_{1}, \phi_{2} \in \mathrm{F}_{2}, \phi_{3} \in \mathrm{F}_{3}, \phi_{4} \in \mathrm{F}_{4}$, and $\psi \in \Phi_{3}$ such that

$$
d(T x, S y) \leq \psi(M(x, y)) \text { for all } x, y \in X
$$

where

$$
\begin{aligned}
M(x, y)= & \max \left\{d(A x, B y), d(A x, T x), d(B y, S y), \phi_{1}(d(A x, S y), d(T x, B y)),\right. \\
& \phi_{2}(d(A x, S y), d(T x, B y), d(A x, B y)), \phi_{3}(d(A x, T x), d(B y, S y), d(A x, B y)) \\
& \left.\phi_{4}(d(A x, S y), d(T x, B y), d(A x, T x), d(B y, S y))\right\} .
\end{aligned}
$$

Then $A, B, S$, and $T$ have a unique common fixed point in $X$.

Proof Let $x_{0} \in X$ be an arbitrary point and let $y_{1}=T x_{0}$. Since $T x_{0} \in T(X) \subseteq B(X)$, there exists $x_{1} \in X$ such that $y_{1}=T x_{0}=B x_{1}$. Let $y_{2}=S x_{1}$. Since $S x_{1} \in S(X) \subseteq A(X)$, there exists $x_{2} \in X$ such that $y_{2}=S x_{1}=A x_{2}$. Let $y_{3}=T x_{2}$. Repeating again and again this process, we can find two sequences $\left\{x_{n}\right\}$ and $\left\{y_{n}\right\}$ in $X$ such that

$$
y_{2 n+1}=B x_{2 n+1}=T x_{2 n} \quad \text { and } \quad y_{2 n+2}=A x_{2 n+2}=S x_{2 n+1} \quad \text { for all } n \in \mathbb{N}_{0} .
$$

Let define $d_{n}=d\left(y_{n}, y_{n+1}\right)$ for all $n \in \mathbb{N}_{0}$. We claim that $\lim _{n \rightarrow \infty} d_{n}=0$. Indeed, by (3) and (5), for all $n \in \mathbb{N}$,

$$
d_{2 n}=d\left(y_{2 n}, y_{2 n+1}\right)=d\left(y_{2 n+1}, y_{2 n}\right)=d\left(T x_{2 n}, S x_{2 n-1}\right) \leq \psi\left(M\left(x_{2 n}, x_{2 n-1}\right)\right) .
$$

Taking into account the properties that define functions in $\left\{\mathrm{F}_{i}\right\}_{i=1}^{4}$, we deduce that

$$
\begin{aligned}
& d\left(A x_{2 n}, B x_{2 n-1}\right)=d\left(y_{2 n}, y_{2 n-1}\right)=d\left(y_{2 n-1}, y_{2 n}\right)=d_{2 n-1} ; \\
& d\left(A x_{2 n}, T x_{2 n}\right)=d\left(y_{2 n}, y_{2 n+1}\right)=d_{2 n} ; \\
& d\left(B x_{2 n-1}, S x_{2 n-1}\right)=d\left(y_{2 n-1}, y_{2 n}\right)=d_{2 n-1} \\
& \phi_{1}\left(d\left(A x_{2 n}, S x_{2 n-1}\right), d\left(T x_{2 n}, B x_{2 n-1}\right)\right) \\
& \quad=\phi_{1}\left(d\left(y_{2 n}, y_{2 n}\right), d\left(y_{2 n+1}, y_{2 n-1}\right)\right)
\end{aligned}
$$




$$
\begin{aligned}
& =\phi_{1}\left(0, d\left(y_{2 n-1}, y_{2 n+1}\right)\right) \stackrel{\left(F_{12}\right)}{\leq} \frac{d\left(y_{2 n-1}, y_{2 n+1}\right)}{2} \\
& \leq \frac{d\left(y_{2 n-1}, y_{2 n}\right)+d\left(y_{2 n}, y_{2 n+1}\right)}{2} \\
& \leq \max \left\{d\left(y_{2 n+1}, y_{2 n}\right), d\left(y_{2 n}, y_{2 n-1}\right)\right\}=\max \left\{d_{2 n-1}, d_{2 n}\right\} \\
\phi_{2} & \left(d\left(A x_{2 n}, S x_{2 n-1}\right), d\left(T x_{2 n}, B x_{2 n-1}\right), d\left(A x_{2 n}, B x_{2 n-1}\right)\right) \\
& =\phi_{2}\left(d\left(y_{2 n}, y_{2 n}\right), d\left(y_{2 n+1}, y_{2 n-1}\right), d\left(y_{2 n}, y_{2 n-1}\right)\right) \\
& =\phi_{2}\left(0, d\left(y_{2 n+1}, y_{2 n-1}\right), d\left(y_{2 n}, y_{2 n-1}\right)\right) \stackrel{\left(F_{22}\right)}{\leq} \max \left\{d_{2 n-1}, d_{2 n}\right\} \\
\phi_{3} & \left(d\left(A x_{2 n}, T x_{2 n}\right), d\left(B x_{2 n-1}, S x_{2 n-1}\right), d\left(A x_{2 n}, B x_{2 n-1}\right)\right) \\
& =\phi_{3}\left(d\left(y_{2 n}, y_{2 n+1}\right), d\left(y_{2 n-1}, y_{2 n}\right), d\left(y_{2 n}, y_{2 n-1}\right)\right) \\
& =\phi_{3}\left(d_{2 n}, d_{2 n-1}, d_{2 n-1}\right) \stackrel{\left(F_{32}\right)}{\leq} \max \left\{d_{2 n-1}, d_{2 n}\right\} \\
\phi_{4} & \left(d\left(A x_{2 n}, S x_{2 n-1}\right), d\left(T x_{2 n}, B x_{2 n-1}\right), d\left(A x_{2 n}, T x_{2 n}\right), d\left(B x_{2 n-1}, S x_{2 n-1}\right)\right) \\
& =\phi_{4}\left(d\left(y_{2 n}, y_{2 n}\right), d\left(y_{2 n+1}, y_{2 n-1}\right), d\left(y_{2 n}, y_{2 n+1}\right), d\left(y_{2 n-1}, y_{2 n}\right)\right) \\
& \leq \phi_{4}\left(0, d\left(y_{2 n+1}, y_{2 n-1}\right), d\left(y_{2 n}, y_{2 n+1}\right), d\left(y_{2 n-1}, y_{2 n}\right)\right) \\
& \left(F_{42}\right) \\
& \leq \max \left\{\frac{d\left(y_{2 n+1}, y_{2 n-1}\right)}{2}, d\left(y_{2 n}, y_{2 n+1}\right), d\left(y_{2 n-1}, y_{2 n}\right)\right\} \\
& \max \left\{d_{2 n-1}, d_{2 n}\right\} . \\
& \\
&
\end{aligned}
$$

Hence,

$$
\begin{aligned}
M\left(x_{2 n}, x_{2 n-1}\right)= & \max \left\{d\left(A x_{2 n}, B x_{2 n-1}\right), d\left(A x_{2 n}, T x_{2 n}\right), d\left(B x_{2 n-1}, S x_{2 n-1}\right)\right. \\
& \phi_{1}\left(d\left(A x_{2 n}, S x_{2 n-1}\right), d\left(T x_{2 n}, B x_{2 n-1}\right)\right) \\
& \phi_{2}\left(d\left(A x_{2 n}, S x_{2 n-1}\right), d\left(T x_{2 n}, B x_{2 n-1}\right), d\left(A x_{2 n}, B x_{2 n-1}\right)\right) \\
& \phi_{3}\left(d\left(A x_{2 n}, T x_{2 n}\right), d\left(B x_{2 n-1}, S x_{2 n-1}\right), d\left(A x_{2 n}, B x_{2 n-1}\right)\right) \\
& \left.\phi_{4}\left(d\left(A x_{2 n}, S x_{2 n-1}\right), d\left(T x_{2 n}, B x_{2 n-1}\right), d\left(A x_{2 n}, T x_{2 n}\right), d\left(B x_{2 n-1}, S x_{2 n-1}\right)\right)\right\} \\
= & \max \left\{d_{2 n-1}, d_{2 n}\right\} .
\end{aligned}
$$

By (6),

$$
d_{2 n} \leq \psi\left(M\left(x_{2 n}, x_{2 n-1}\right)\right)=\psi\left(\max \left\{d_{2 n-1}, d_{2 n}\right\}\right)
$$

If $d_{2 n-1}<d_{2 n}$ for some $n$, then $d_{2 n}>0$ and we get the contradiction $d_{2 n} \leq \psi\left(\max \left\{d_{2 n-1}\right.\right.$, $\left.\left.d_{2 n}\right\}\right)=\psi\left(d_{2 n}\right)$ (recall Lemma 4 ). As a consequence, we deduce that $d_{2 n} \leq d_{2 n-1}$ for all $n \in \mathbb{N}$. Similarly, it can be proved that $d_{2 n+1} \leq \psi\left(\left\{d_{2 n}, d_{2 n+1}\right\}\right)$, so $d_{2 n+1} \leq d_{2 n}$ for all $n \in \mathbb{N}$. Hence,

$$
d_{n+1} \leq \psi\left(d_{n}\right) \quad \text { and } \quad d_{n+1} \leq d_{n} \quad \text { for all } n \in \mathbb{N}
$$


Since $\left\{d_{n}\right\}$ is a non-increasing sequence of non-negative real numbers, it is convergent. Let $L \geq 0$ be its limit. Taking into account that $\psi \in \Phi_{3}$ and $d_{n+1} \leq \psi\left(d_{n}\right)$ for all $n \in \mathbb{N}$, we deduce that $\left\{d_{n}\right\} \rightarrow 0$, that is,

$$
\lim _{n \rightarrow \infty} d\left(y_{n}, y_{n+1}\right)=0 .
$$

Next, let us show that $\left\{y_{n}\right\}$ is a Cauchy sequence in $(X, d)$ reasoning by contradiction. Assume that $\left\{y_{n}\right\}$ is not a Cauchy sequence. By Proposition 5, the sequence $\left\{y_{2 n}\right\}$ is not a Cauchy sequence. In particular, there exist $\varepsilon_{0}>0$ and two subsequences $\left\{y_{2 n(k)}\right\}$ and $\left\{y_{2 m(k)}\right\}$ of $\left\{y_{2 n}\right\}$ such that

$$
\begin{aligned}
2 k<2 n(k)<2 m(k), & d\left(y_{2 n(k)}, y_{2 m(k)-2}\right) \leq \varepsilon_{0}<d\left(y_{2 n(k)}, y_{2 m(k)}\right) \quad \text { for all } k \in \mathbb{N}, \\
\lim _{n \rightarrow \infty} d\left(y_{2 n(k)}, y_{2 m(k)}\right)= & \lim _{n \rightarrow \infty} d\left(y_{2 n(k)}, y_{2 m(k)-1}\right)=\lim _{n \rightarrow \infty} d\left(y_{2 n(k)}, y_{2 m(k)+1}\right) \\
= & \lim _{n \rightarrow \infty} d\left(y_{2 n(k)-1}, y_{2 m(k)-1}\right)=\lim _{n \rightarrow \infty} d\left(y_{2 n(k)-1}, y_{2 m(k)+1}\right)=\varepsilon_{0} .
\end{aligned}
$$

By (3) and (5), for all $k \in \mathbb{N}$,

$$
d\left(y_{2 m(k)+1}, y_{2 n(k)}\right)=d\left(T x_{2 m(k)}, S x_{2 n(k)-1}\right) \leq \psi\left(M\left(x_{2 m(k)}, x_{2 n(k)-1}\right)\right) .
$$

Notice that

$$
\begin{aligned}
& \lim _{k \rightarrow \infty} d\left(A x_{2 m(k)}, B x_{2 n(k)-1}\right)=\lim _{k \rightarrow \infty} d\left(y_{2 m(k)}, y_{2 n(k)-1}\right)=\varepsilon_{0} ; \\
& \lim _{k \rightarrow \infty} d\left(A x_{2 m(k)}, T x_{2 m(k)}\right)=\lim _{k \rightarrow \infty} d\left(y_{2 m(k)}, y_{2 m(k)+1}\right)=0 ; \\
& \lim _{k \rightarrow \infty} d\left(B x_{2 n(k)-1}, S x_{2 n(k)-1}\right)=\lim _{k \rightarrow \infty} d\left(y_{2 n(k)-1}, y_{2 n(k)}\right)=0 ; \\
& \lim _{k \rightarrow \infty} \phi_{1}\left(d\left(A x_{2 m(k)}, S x_{2 n(k)-1}\right), d\left(T x_{2 m(k)}, B x_{2 n(k)-1}\right)\right) \\
& \quad=\lim _{k \rightarrow \infty} \phi_{1}\left(d\left(y_{2 m(k)}, y_{2 n(k)}\right), d\left(y_{2 m(k)+1}, y_{2 n(k)-1}\right)\right) \\
& \quad \stackrel{\left(F_{11}\right)}{=} \phi_{1}\left(\lim _{k \rightarrow \infty} d\left(y_{2 m(k)}, y_{2 n(k)}\right), \lim _{k \rightarrow \infty} d\left(y_{2 m(k)+1}, y_{2 n(k)-1}\right)\right)=\phi_{1}\left(\varepsilon_{0}, \varepsilon_{0}\right) \stackrel{\left(F_{13}\right)}{\leq} \varepsilon_{0} ; \\
& \lim _{k \rightarrow \infty} \phi_{2}\left(d\left(A x_{2 m(k)}, S x_{2 n(k)-1}\right), d\left(T x_{2 m(k)}, B x_{2 n(k)-1}\right), d\left(A x_{2 m(k)}, B x_{2 n(k)-1}\right)\right) \\
& \quad=\lim _{k \rightarrow \infty} \phi_{2}\left(d\left(y_{2 m(k)}, y_{2 n(k)}\right), d\left(y_{2 m(k)+1}, y_{2 n(k)-1}\right), d\left(y_{2 m(k)}, y_{2 n(k)-1}\right)\right) \\
& \quad \stackrel{\left(F_{21}\right)}{=} \phi_{2}\left(\lim _{k \rightarrow \infty} d\left(y_{2 m(k)}, y_{2 n(k)}\right), \lim _{k \rightarrow \infty} d\left(y_{2 m(k)+1}, y_{2 n(k)-1}\right), \lim _{k \rightarrow \infty} d\left(y_{2 m(k)}, y_{2 n(k)-1}\right)\right) \\
& =\phi_{2}\left(\varepsilon_{0}, \varepsilon_{0}, \varepsilon_{0}\right) \stackrel{\left(F_{23}\right)}{\leq} \varepsilon_{0} ; \\
& \lim _{k \rightarrow \infty} \phi_{3}\left(d\left(A x_{2 m(k)}, T x_{2 m(k)}\right), d\left(B x_{2 n(k)-1}, S x_{2 n(k)-1}\right), d\left(A x_{2 m(k)}, B x_{2 n(k)-1}\right)\right) \\
& =\lim _{k \rightarrow \infty} \phi_{3}\left(d\left(y_{2 m(k)}, y_{2 m(k)+1}\right), d\left(y_{2 n(k)-1}, y_{2 n(k)}\right), d\left(y_{2 m(k)}, y_{2 n(k)-1}\right)\right) \\
& \quad \stackrel{\left(F_{31}\right)}{=} \phi_{3}\left(\lim _{k \rightarrow \infty} d\left(y_{2 m(k)}, y_{2 m(k)+1}\right), \lim _{k \rightarrow \infty} d\left(y_{2 n(k)-1}, y_{2 n(k)}\right), \lim _{k \rightarrow \infty} d\left(y_{2 m(k)}, y_{2 n(k)-1}\right)\right) \\
& =\phi_{3}\left(0,0, \varepsilon_{0}\right) \stackrel{\left(F_{33}\right)}{\leq} \varepsilon_{0} ;
\end{aligned}
$$




$$
\begin{aligned}
\lim _{k \rightarrow \infty} & \phi_{4}\left(d\left(A x_{2 m(k)}, S x_{2 n(k)-1}\right), d\left(T x_{2 m(k)}, B x_{2 n(k)-1}\right), d\left(A x_{2 m(k)}, T x_{2 m(k)}\right),\right. \\
& \left.d\left(B x_{2 n(k)-1}, S x_{2 n(k)-1}\right)\right) \\
= & \lim _{k \rightarrow \infty} \phi_{4}\left(d\left(y_{2 m(k)}, y_{2 n(k)}\right), d\left(y_{2 m(k)+1}, y_{2 n(k)-1}\right), d\left(y_{2 m(k)}, y_{2 m(k)+1}\right), d\left(y_{2 n(k)-1}, y_{2 n(k)}\right)\right) \\
& \stackrel{\left(\mathrm{F}_{41}\right)}{=} \phi_{4}\left(\lim _{k \rightarrow \infty} d\left(y_{2 m(k)}, y_{2 n(k)}\right), \lim _{k \rightarrow \infty} d\left(y_{2 m(k)+1}, y_{2 n(k)-1}\right),\right. \\
& \left.\lim _{k \rightarrow \infty} d\left(y_{2 m(k)}, y_{2 m(k)+1}\right), \lim _{k \rightarrow \infty} d\left(y_{2 n(k)-1}, y_{2 n(k)}\right)\right)=\phi_{4}\left(\varepsilon_{0}, \varepsilon_{0}, 0,0\right) \stackrel{\left(F_{43}\right)}{\leq} \varepsilon_{0} .
\end{aligned}
$$

As a result, it follows from (8) and (7),

$$
\begin{aligned}
& \lim _{k \rightarrow \infty} M\left(x_{2 m(k)}, x_{2 n(k)-1}\right) \\
&=\lim _{k \rightarrow \infty} \max \left\{d\left(A x_{2 m(k)}, B x_{2 n(k)-1}\right), d\left(A x_{2 m(k)}, T x_{2 m(k)}\right), d\left(B x_{2 n(k)-1}, S x_{2 n(k)-1}\right),\right. \\
& \quad \phi_{1}\left(d\left(A x_{2 m(k)}, S x_{2 n(k)-1}\right), d\left(T x_{2 m(k)}, B x_{2 n(k)-1}\right)\right), \\
& \quad \phi_{2}\left(d\left(A x_{2 m(k)}, S x_{2 n(k)-1}\right), d\left(T x_{2 m(k)}, B x_{2 n(k)-1}\right), d\left(A x_{2 m(k)}, B x_{2 n(k)-1}\right)\right), \\
& \quad \phi_{3}\left(d\left(A x_{2 m(k)}, T x_{2 m(k)}\right), d\left(B x_{2 n(k)-1}, S x_{2 n(k)-1}\right), d\left(A x_{2 m(k)}, B x_{2 n(k)-1}\right)\right), \\
& \phi_{4}\left(d\left(A x_{2 m(k)}, S x_{2 n(k)-1}\right), d\left(T x_{2 m(k)}, B x_{2 n(k)-1}\right),\right. \\
&\left.\left.d\left(A x_{2 m(k)}, T x_{2 m(k)}\right), d\left(B x_{2 n(k)-1}, S x_{2 n(k)-1}\right)\right)\right\} \\
&= \varepsilon_{0} .
\end{aligned}
$$

As $\psi$ is upper semi-continuous, we deduce that

$$
\begin{aligned}
\varepsilon_{0} & =\lim _{n \rightarrow \infty} d\left(y_{2 m(k)+1}, y_{2 n(k)}\right)=\limsup _{n \rightarrow \infty} d\left(y_{2 m(k)+1}, y_{2 n(k)}\right) \\
& \leq \limsup _{n \rightarrow \infty} \psi\left(M\left(x_{2 m(k)}, x_{2 n(k)-1}\right)\right) \leq \psi\left(\varepsilon_{0}\right)<\varepsilon_{0},
\end{aligned}
$$

which is a contradiction. As a result, $\left\{y_{n}\right\}$ is a Cauchy sequence in $(X, d)$. Then there exists $z \in X$ such that $\left\{y_{n}\right\} \rightarrow z$. In particular,

$$
z=\lim _{n \rightarrow \infty} y_{n}=\lim _{n \rightarrow \infty} T x_{2 n}=\lim _{n \rightarrow \infty} B x_{2 n+1}=\lim _{n \rightarrow \infty} A x_{2 n}=\lim _{n \rightarrow \infty} S x_{2 n-1}
$$

Next we distinguish some cases depending on the complete set. Notice that

$$
\begin{aligned}
& \left\{y_{2 n+2}=A x_{2 n+2}=S x_{2 n+1}\right\} \subseteq S(X) \subseteq A(X) \text { and } \\
& \left\{y_{2 n+1}=B x_{2 n+1}=T x_{2 n}\right\} \subseteq T(X) \subseteq B(X) .
\end{aligned}
$$

Assume that $A(X)$ (or $S(X))$ is complete. Then there exists $u \in X$ such that $z=A u$. To show that $T u=z$, suppose, by contradiction, that $d(T u, z)>0$. Therefore, for all $n \in \mathbb{N}$,

$$
d\left(T u, y_{2 n+2}\right)=d\left(T u, S x_{2 n+1}\right) \leq \psi\left(M\left(u, x_{2 n+1}\right)\right) .
$$


Notice that

$$
\begin{aligned}
& \lim _{n \rightarrow \infty} d\left(A u, B x_{2 n+1}\right)=\lim _{n \rightarrow \infty} d\left(z, y_{2 n+1}\right)=0 ; \\
& \lim _{n \rightarrow \infty} d(A u, T u)=d(z, T u)>0 ; \\
& \lim _{n \rightarrow \infty} d\left(B x_{2 n+1}, S x_{2 n+1}\right)=d\left(y_{2 n+1}, y_{2 n+2}\right)=0 ; \\
& \lim _{n \rightarrow \infty} \phi_{1}\left(d\left(A u, S x_{2 n+1}\right), d\left(T u, B x_{2 n+1}\right)\right) \\
& =\lim _{n \rightarrow \infty} \phi_{1}\left(d\left(z, y_{2 n+2}\right), d\left(T u, y_{2 n+1}\right)\right) \\
& \stackrel{\left(F_{11}\right)}{=} \phi_{1}\left(\lim _{n \rightarrow \infty} d\left(z, y_{2 n+2}\right), \lim _{n \rightarrow \infty} d\left(T u, y_{2 n+1}\right)\right) \\
& =\phi_{1}(0, d(T u, z)) \stackrel{\left(F_{12}\right)}{\leq} \frac{d(T u, z)}{2}<d(T u, z) ; \\
& \lim _{n \rightarrow \infty} \phi_{2}\left(d\left(A u, S x_{2 n+1}\right), d\left(T u, B x_{2 n+1}\right), d\left(A u, B x_{2 n+1}\right)\right) \\
& =\lim _{n \rightarrow \infty} \phi_{2}\left(d\left(z, y_{2 n+2}\right), d\left(T u, y_{2 n+1}\right), d\left(z, y_{2 n+1}\right)\right) \\
& \stackrel{\left(F_{21}\right)}{=} \phi_{2}\left(\lim _{n \rightarrow \infty} d\left(z, y_{2 n+2}\right), \lim _{n \rightarrow \infty} d\left(T u, y_{2 n+1}\right), \lim _{n \rightarrow \infty} d\left(z, y_{2 n+1}\right)\right) \\
& =\phi_{2}(0, d(T u, z), 0) \stackrel{\left(F_{22}\right)}{\leq} d(T u, z) \text {; } \\
& \lim _{n \rightarrow \infty} \phi_{3}\left(d(A u, T u), d\left(B x_{2 n+1}, S x_{2 n+1}\right), d\left(A u, B x_{2 n+1}\right)\right) \\
& =\lim _{n \rightarrow \infty} \phi_{3}\left(d(z, T u), d\left(y_{2 n+1}, y_{2 n+2}\right), d\left(z, y_{2 n+1}\right)\right) \\
& \stackrel{\left(\mathrm{F}_{31}\right)}{=} \phi_{3}\left(\lim _{n \rightarrow \infty} d(z, T u), \lim _{n \rightarrow \infty} d\left(y_{2 n+1}, y_{2 n+2}\right), \lim _{n \rightarrow \infty} d\left(z, y_{2 n+1}\right)\right) \\
& =\phi_{3}(d(z, T u), 0,0) \stackrel{\left(F_{32}\right)}{\leq} d(z, T u) \text {; } \\
& \lim _{n \rightarrow \infty} \phi_{4}\left(d\left(A u, S x_{2 n+1}\right), d\left(T u, B x_{2 n+1}\right), d(A u, T u), d\left(B x_{2 n+1}, S x_{2 n+1}\right)\right) \\
& =\lim _{n \rightarrow \infty} \phi_{4}\left(d\left(z, y_{2 n+2}\right), d\left(T u, y_{2 n+1}\right), d(z, T u), d\left(y_{2 n+1}, y_{2 n+2}\right)\right) \\
& \stackrel{\left(\mathrm{F}_{41}\right)}{=} \phi_{4}\left(\lim _{n \rightarrow \infty} d\left(z, y_{2 n+2}\right), \lim _{n \rightarrow \infty} d\left(T u, y_{2 n+1}\right), \lim _{n \rightarrow \infty} d(z, T u), \lim _{n \rightarrow \infty} d\left(y_{2 n+1}, y_{2 n+2}\right)\right) \\
& =\phi_{4}(0, d(T u, z), d(z, T u), 0) \stackrel{\left(F_{42}\right)}{\leq} d(z, T u) \text {. }
\end{aligned}
$$

Since

$$
\begin{aligned}
& \lim _{n \rightarrow \infty} M\left(u, x_{2 n+1}\right) \\
&=\lim _{n \rightarrow \infty} \max \left\{d\left(A u, B x_{2 n+1}\right), d(A u, T u), d\left(B x_{2 n+1}, S x_{2 n+1}\right),\right. \\
& \phi_{1}\left(d\left(A u, S x_{2 n+1}\right), d\left(T u, B x_{2 n+1}\right)\right), \phi_{2}\left(d\left(A u, S x_{2 n+1}\right), d\left(T u, B x_{2 n+1}\right), d\left(A u, B x_{2 n+1}\right)\right), \\
& \phi_{3}\left(d(A u, T u), d\left(B x_{2 n+1}, S x_{2 n+1}\right), d\left(A u, B x_{2 n+1}\right)\right), \\
&\left.\phi_{4}\left(d\left(A u, S x_{2 n+1}\right), d\left(T u, B x_{2 n+1}\right), d(A u, T u), d\left(B x_{2 n+1}, S x_{2 n+1}\right)\right)\right\} \\
&= d(z, T u)>0
\end{aligned}
$$


letting $n \rightarrow \infty$ in (9), the upper semi-continuity of $\psi$ yields

$$
\begin{aligned}
d(z, T u) & =\limsup _{n \rightarrow \infty} d\left(T u, y_{2 n+2}\right) \leq \limsup _{n \rightarrow \infty} \psi\left(M\left(u, x_{2 n+1}\right)\right) \\
& \leq \psi\left(\limsup _{n \rightarrow \infty} M\left(u, x_{2 n+1}\right)\right)=\psi(d(z, T u))<d(z, T u),
\end{aligned}
$$

which is a contradiction. As a consequence, $T u=z=A u$. As $T(X) \subseteq B(X)$, there exists $w \in X$ such that $T u=B w$. Then $A u=T u=z=B w$. We claim that $z=S w$. To prove it, suppose that $d(z, S w)>0$. By (3), for all $n \in \mathbb{N}$,

$$
d\left(y_{2 n+1}, S w\right)=d\left(T x_{2 n}, S w\right) \leq \psi\left(M\left(x_{2 n}, w\right)\right) .
$$

Notice that

$$
\begin{aligned}
& \lim _{n \rightarrow \infty} d\left(A x_{2 n}, B w\right)=\lim _{n \rightarrow \infty} d\left(y_{2 n}, z\right)=0 ; \\
& \lim _{n \rightarrow \infty} d\left(A x_{2 n}, T x_{2 n}\right)=\lim _{n \rightarrow \infty} d\left(y_{2 n}, y_{2 n+1}\right)=0 \text {; } \\
& \lim _{n \rightarrow \infty} d(B w, S w)=d(z, S w)>0 ; \\
& \lim _{n \rightarrow \infty} \phi_{1}\left(d\left(A x_{2 n}, S w\right), d\left(T x_{2 n}, B w\right)\right) \\
& =\lim _{n \rightarrow \infty} \phi_{1}\left(d\left(y_{2 n}, S w\right), d\left(y_{2 n+1}, z\right)\right) \\
& \stackrel{\left(F_{11}\right)}{=} \phi_{1}\left(\lim _{n \rightarrow \infty} d\left(y_{2 n}, S w\right), \lim _{n \rightarrow \infty} d\left(y_{2 n+1}, z\right)\right) \\
& =\phi_{1}(d(z, S w), 0) \stackrel{\left(F_{14}\right)}{\leq} d(z, S w) ; \\
& \lim _{n \rightarrow \infty} \phi_{2}\left(d\left(A x_{2 n}, S w\right), d\left(T x_{2 n}, B w\right), d\left(A x_{2 n}, B w\right)\right) \\
& =\lim _{n \rightarrow \infty} \phi_{2}\left(d\left(y_{2 n}, S w\right), d\left(y_{2 n+1}, z\right), d\left(y_{2 n}, z\right)\right) \\
& \stackrel{\left(F_{21}\right)}{=} \phi_{2}\left(\lim _{n \rightarrow \infty} d\left(y_{2 n}, S w\right), \lim _{n \rightarrow \infty} d\left(y_{2 n+1}, z\right), \lim _{n \rightarrow \infty} d\left(y_{2 n}, z\right)\right) \\
& =\phi_{2}(d(z, S w), 0,0) \stackrel{\left(F_{24}\right)}{\leq} d(z, S w) ; \\
& \lim _{n \rightarrow \infty} \phi_{3}\left(d\left(A x_{2 n}, T x_{2 n}\right), d(B w, S w), d\left(A x_{2 n}, B w\right)\right) \\
& =\lim _{n \rightarrow \infty} \phi_{3}\left(d\left(y_{2 n}, y_{2 n+1}\right), d(z, S w), d\left(y_{2 n}, z\right)\right) \\
& \stackrel{\left(\mathrm{F}_{31}\right)}{=} \phi_{3}\left(\lim _{n \rightarrow \infty} d\left(y_{2 n}, y_{2 n+1}\right), \lim _{n \rightarrow \infty} d(z, S w), \lim _{n \rightarrow \infty} d\left(y_{2 n}, z\right)\right) \\
& =\phi_{3}(0, d(z, S w), 0) \stackrel{\left(F_{34}\right)}{\leq} d(z, S w) ; \\
& \lim _{n \rightarrow \infty} \phi_{4}\left(d\left(A x_{2 n}, S w\right), d\left(T x_{2 n}, B w\right), d\left(A x_{2 n}, T x_{2 n}\right), d(B w, S w)\right) \\
& =\lim _{n \rightarrow \infty} \phi_{4}\left(d\left(y_{2 n}, S w\right), d\left(y_{2 n+1}, z\right), d\left(y_{2 n}, y_{2 n+1}\right), d(z, S w)\right) \\
& \stackrel{\left(F_{41}\right)}{=} \phi_{4}\left(\lim _{n \rightarrow \infty} d\left(y_{2 n}, S w\right), \lim _{n \rightarrow \infty} d\left(y_{2 n+1}, z\right), \lim _{n \rightarrow \infty} d\left(y_{2 n}, y_{2 n+1}\right), \lim _{n \rightarrow \infty} d(z, S w)\right) \\
& =\phi_{4}(d(z, S w), 0,0, d(z, S w)) \stackrel{\left(F_{44}\right)}{\leq} d(z, S w) \text {. }
\end{aligned}
$$


Hence,

$$
\begin{aligned}
\lim _{n \rightarrow \infty} M\left(x_{2 n}, w\right)= & \lim _{n \rightarrow \infty} \max \left\{d\left(A x_{2 n}, B w\right), d\left(A x_{2 n}, T x_{2 n}\right), d(B w, S w),\right. \\
& \phi_{1}\left(d\left(A x_{2 n}, S w\right), d\left(T x_{2 n}, B w\right)\right), \\
& \phi_{2}\left(d\left(A x_{2 n}, S w\right), d\left(T x_{2 n}, B w\right), d\left(A x_{2 n}, B w\right)\right), \\
& \phi_{3}\left(d\left(A x_{2 n}, T x_{2 n}\right), d(B w, S w), d\left(A x_{2 n}, B w\right)\right), \\
& \left.\phi_{4}\left(d\left(A x_{2 n}, S w\right), d\left(T x_{2 n}, B w\right), d\left(A x_{2 n}, T x_{2 n}\right), d(B w, S w)\right)\right\} \\
= & d(z, S w)>0 .
\end{aligned}
$$

Again, letting $n \rightarrow \infty$ in (10) and using the upper semi-continuity of $\psi$, we deduce that

$$
\begin{aligned}
d(z, S w) & =\limsup _{n \rightarrow \infty} d\left(y_{2 n+1}, S w\right) \leq \limsup _{n \rightarrow \infty} \psi\left(M\left(x_{2 n}, w\right)\right) \\
& \leq \psi\left(\lim _{n \rightarrow \infty} M\left(x_{2 n}, w\right)\right)=\psi(d(z, S w))<d(z, S w)
\end{aligned}
$$

which is a contradiction. Thus, $S w=z$, which means that $z=T u=A u=B w=S w$. As $\{A, T\}$ and $\{B, S\}$ are weakly compatible,

$$
A z=A T u=T A u=T z \quad \text { and } \quad B z=B S w=S B w=S z
$$

Next, let us show that $T z=S z$. On the contrary, suppose that $d(T z, S z)>0$. Hence, by (3),

$$
d(T z, S z) \leq \psi(M(z, z))
$$

Notice that

$$
\begin{aligned}
& d(A z, B z)=d(T z, S z)>0 ; \\
& d(A z, T z)=d(T z, T z)=0 \\
& d(B z, S z)=d(S z, S z)=0 ; \\
& \phi_{1}(d(A z, S z), d(T z, B z))=\phi_{1}(d(T z, S z), d(T z, S z)) \stackrel{\left(F_{13}\right)}{\leq} d(T z, S z) ; \\
& \phi_{2}(d(A z, S z), d(T z, B z), d(A z, B z)) \\
& \quad=\phi_{2}(d(T z, S z), d(T z, S z), d(T z, S z)) \stackrel{\left(F_{23}\right)}{\leq} d(T z, S z) ; \\
& \quad \phi_{3}(d(A z, T z), d(B z, S z), d(A z, B z)) \\
& \quad=\phi_{3}(d(T z, T z), d(S z, S z), d(T z, S z)) \\
& \quad=\phi_{3}(0,0, d(T z, S z)) \stackrel{\left(F_{33}\right)}{\leq} d(T z, S z) ; \\
& \phi_{4}(d(A z, S z), d(T z, B z), d(A z, T z), d(B z, S z)) \\
& \quad=\phi_{4}(d(T z, S z), d(T z, S z), d(T z, T z), d(S z, S z)) \\
& \quad=\phi_{4}(d(T z, S z), d(T z, S z), 0,0) \stackrel{\left(F_{43}\right)}{\leq} d(T z, S z) .
\end{aligned}
$$


Therefore,

$$
\begin{aligned}
M(z, z)= & \max \{d(A z, B z), d(A z, T z), d(B z, S z) \\
& \phi_{1}(d(A z, S z), d(T z, B z)), \phi_{2}(d(A z, S z), d(T z, B z), d(A z, B z)) \\
& \phi_{3}(d(A z, T z), d(B z, S z), d(A z, B z)) \\
& \left.\phi_{4}(d(A z, S z), d(T z, B z), d(A z, T z), d(B z, S z))\right\} \\
= & d(T z, S z)>0
\end{aligned}
$$

Again, it follows from (11) that

$$
d(T z, S z) \leq \psi(M(z, z))=\psi(d(T z, S z))<d(T z, S z)
$$

which is a contradiction. As a consequence, we conclude that $T z=S z$, which means that

$$
A z=T z=S z=B z
$$

In particular, $z$ is a coincidence point of $A, B, T$, and $S$.

Next, let us show that $T z=z$. Reasoning by contradiction, assume that $d(T z, z)>0$. By (3),

$$
d(T z, z)=d(T z, S w) \leq \psi(M(z, w)) .
$$

Notice that

$$
\begin{aligned}
& d(A z, B w)=d(T z, S w)=d(T z, z)>0 ; \\
& d(A z, T z)=d(T z, T z)=0 ; \\
& d(B w, S w)=d(S w, S w)=0 ; \\
& \phi_{1}(d(A z, S w), d(T z, B w))=\phi_{1}(d(T z, S w), d(T z, S w)) \stackrel{\left(F_{13}\right)}{\leq} d(T z, z) ; \\
& \phi_{2}(d(A z, S w), d(T z, B w), d(A z, B w))=\phi_{2}(d(T z, z), d(T z, z), d(T z, z)) \stackrel{\left(F_{23}\right)}{\leq} d(T z, z) ; \\
& \phi_{3}(d(A z, T z), d(B w, S w), d(A z, B w)) \\
& \quad=\phi_{3}(d(T z, T z), d(z, z), d(T z, z))=\phi_{3}(0,0, d(T z, z)) \stackrel{\left(F_{33}\right)}{\leq} d(T z, z) ; \\
& \phi_{4}(d(A z, S w), d(T z, B w), d(A z, T z), d(B w, S w)) \\
& \quad=\phi_{4}(d(T z, z), d(T z, z), d(T z, T z), d(z, z))=\phi_{4}(d(T z, z), d(T z, z), 0,0) \stackrel{\left(F_{43}\right)}{\leq} d(T z, z) .
\end{aligned}
$$

As a result,

$$
\begin{aligned}
M(z, w)= & \max \left\{d(A z, B w), d(A z, T z), d(B w, S w), \phi_{1}(d(A z, S w), d(T z, B w)),\right. \\
& \phi_{2}(d(A z, S w), d(T z, B w), d(A z, B w)), \phi_{3}(d(A z, T z), d(B w, S w), d(A z, B w)) \\
& \left.\phi_{4}(d(A z, S w), d(T z, B w), d(A z, T z), d(B w, S w))\right\}=d(T z, z)>0 .
\end{aligned}
$$


Again, it follows from (12) that

$$
d(T z, z) \leq \psi(M(z, w))<d(T z, z)
$$

which is a contradiction. Thus, $T z=z$, so $z$ is a common fixed point of $A, B, T$, and $S$.

Finally, let us show that $z$ is the unique common fixed point of $A, T, S$, and $B$. Assume that $x, y \in X$ are two different common fixed points of $A, T, S$, and $B$. Then $d(x, y)>0$. Notice that

$$
\begin{aligned}
& d(A x, B y)=d(x, y)>0 ; \\
& d(A x, T x)=d(x, x)=0 ; \\
& d(B y, S y)=d(y, y)=0 ; \\
& \phi_{1}(d(A x, S y), d(T x, B y))=\phi_{1}(d(x, y), d(x, y)) \stackrel{\left(F_{13}\right)}{\leq} d(x, y) ; \\
& \phi_{2}(d(A x, S y), d(T x, B y), d(A x, B y))=\phi_{2}(d(x, y), d(x, y), d(x, y)) \stackrel{\left(F_{23}\right)}{\leq} d(x, y) ; \\
& \phi_{3}(d(A x, T x), d(B y, S y), d(A x, B y)) \\
& \quad=\phi_{3}(d(x, x), d(y, y), d(x, y))=\phi_{3}(0,0, d(x, y)) \stackrel{\left(F_{33}\right)}{\leq} d(x, y) ; \\
& \phi_{4}(d(A x, S y), d(T x, B y), d(A x, T x), d(B y, S y)) \\
& \quad=\phi_{4}(d(x, y), d(x, y), d(x, x), d(y, y))=\phi_{4}(d(x, y), d(x, y), 0,0) \stackrel{\left(F_{43}\right)}{\leq} d(x, y) .
\end{aligned}
$$

Therefore,

$$
\begin{aligned}
M(x, y)= & \max \left\{d(A x, B y), d(A x, T x), d(B y, S y), \phi_{1}(d(A x, S y), d(T x, B y)),\right. \\
& \phi_{2}(d(A x, S y), d(T x, B y), d(A x, B y)), \phi_{3}(d(A x, T x), d(B y, S y), d(A x, B y)) \\
& \left.\phi_{4}(d(A x, S y), d(T x, B y), d(A x, T x), d(B y, S y))\right\} \\
= & d(x, y)>0 .
\end{aligned}
$$

As a result,

$$
d(x, y)=d(T x, S y) \leq \psi(M(x, y))<d(x, y)
$$

which is a contradiction. Then $x=y$ and $A, B, S$, and $T$ have a unique common fixed point in $X$.

Using whatever functions $\phi_{1} \in F_{1}, \phi_{2} \in F_{2}, \phi_{3} \in F_{3}$, and $\phi_{4} \in F_{4}$ (as we have shown in the first part of this section), we can obtain a large variety of different corollaries. For instance, the following one.

Corollary 7 Theorem 1 immediately follows from Theorem 6.

Proof It is only necessary to take, for all $r, s, t, u \in[0, \infty)$,

$$
\phi_{1}(r, s)=\frac{r+s}{2}, \quad \phi_{2}(r, s, t)=\phi_{3}(r, s, t,)=\frac{r s}{1+t}, \quad \text { and } \quad \phi_{4}(r, s, t, u)=\frac{1+r+s}{1+t+u} t .
$$


Hence $\phi_{1} \in \mathrm{F}_{1}, \phi_{2} \in \mathrm{F}_{2}, \phi_{3} \in \mathrm{F}_{3}$, and $\phi_{4} \in \mathrm{F}_{4}$. Furthermore, for all $x, y \in X$,

$$
\begin{aligned}
& \phi_{1}(d(A x, S y), d(T x, B y))=\frac{d(A x, S y)+d(T x, B y)}{2}, \\
& \phi_{2}(d(A x, S y), d(T x, B y), d(A x, B y))=\frac{d(A x, S y) d(T x, B y)}{1+d(A x, B y)}, \\
& \phi_{3}(d(A x, T x), d(B y, S y), d(A x, B y))=\frac{d(A x, T x) d(B y, S y)}{1+d(A x, B y)}, \quad \text { and } \\
& \phi_{4}(d(A x, S y), d(T x, B y), d(A x, T x), d(B y, S y))=\frac{1+d(A x, S y)+d(T x, B y)}{1+d(A x, T x)+d(B y, S y)} d(A x, T x) .
\end{aligned}
$$

As a result, the contractivity condition (1) implies (3), and Theorem 6 is applicable.

As a particular case, we highlight the situation in which $T=B$ and $S=A$. In such a case, we obtain the following consequence.

Corollary 8 Let $T, S: X \rightarrow X$ be self-mappings in a metric space $(X, d)$ such that

(a) $\{T, S\}$ is weakly compatible;

(b) one of $T(X)$ or $S(X)$ is complete.

Also assume that there exist $\phi_{1} \in \mathrm{F}_{1}, \phi_{2} \in \mathrm{F}_{2}, \phi_{3} \in \mathrm{F}_{3}, \phi_{4} \in \mathrm{F}_{4}$, and $\psi \in \Phi_{3}$ such that

$$
d(T x, S y) \leq \psi(M(x, y)) \quad \text { for all } x, y \in X
$$

where

$$
\begin{aligned}
M(x, y)= & \max \left\{d(S x, T y), d(S x, T x), d(T y, S y), \phi_{1}(d(S x, S y), d(T x, T y)),\right. \\
& \phi_{2}(d(S x, S y), d(T x, T y), d(S x, T y)), \phi_{3}(d(S x, T x), d(T y, S y), d(S x, T y)) \\
& \left.\phi_{4}(d(S x, S y), d(T x, T y), d(S x, T x), d(T y, S y))\right\} .
\end{aligned}
$$

Then $T$ and S have a unique common fixed point in $X$.

The following example shows that Theorem 6 improves Theorem 1. It is based on Example 2.3 in [34].

Example 9 Let $X=[0,1]$ be endowed with the Euclidean metric $d(x, y)=|x-y|$ for all $x, y \in X$, and let $A, B, S, T: X \rightarrow X$ be the self-mappings given, for all $x \in X$, by

$$
A x=x^{2}, \quad B x=\frac{x^{2}}{2}, \quad S x=0, \quad T x= \begin{cases}0, & \text { if } x \in[0,1) \\ 0.9, & \text { if } x=1 .\end{cases}
$$

Notice that, for all $x, y \in X$,

$$
d(T x, S y)= \begin{cases}0.9, & \text { if } x=1 \\ 0, & \text { otherwise }\end{cases}
$$


If $x<1$, the contractivity condition

$$
d(T x, S y) \leq \psi(M(x, y)) \text { for all } y \in X,
$$

is obvious whatever $\psi \in \Phi_{3}$. Next, let $\psi:[0, \infty) \rightarrow[0, \infty)$ and $\phi_{1}:[0, \infty)^{2} \rightarrow[0, \infty)$ be the functions given by

$$
\psi(r)=0.9 r \quad \text { and } \quad \phi_{1}(r, s)=\max \left\{r, \frac{r+s}{2}\right\} \quad \text { for all } r, s \geq 0 .
$$

Clearly, $\psi \in \Phi_{3}$ and $\phi_{1} \in \mathrm{F}_{1}$. Notice that if $x=1$, then $d(A x, S y)=d(1,0)=1$, so $\phi_{1}(d(A x, S y)$, $d(T x, B y))=1$. Hence, for $x=1$,

$$
\begin{aligned}
M(x, y)= & \max \left\{d(A x, B y), d(A x, T x), d(B y, S y), \phi_{1}(d(A x, S y), d(T x, B y)),\right. \\
& \phi_{2}(d(A x, S y), d(T x, B y), d(A x, B y)), \phi_{3}(d(A x, T x), d(B y, S y), d(A x, B y)) \\
& \left.\phi_{4}(d(A x, S y), d(T x, B y), d(A x, T x), d(B y, S y))\right\} \geq 1 .
\end{aligned}
$$

In such a case, for all $y \in X$,

$$
d(T(1), S y)=0.9=\psi(1) \leq \psi(M(1, y))
$$

which means that the contractivity condition (3) holds for all $x, y \in X$. The pairs $\{A, T\}$ and $\{B, S\}$ are weakly compatible because the unique solution of equations $A x=T x$ and $B x=S x$ is $x=0$. As all hypotheses of Theorem 6 are satisfied, we conclude that $A, B, S$, and $T$ have a unique common fixed point in $X$.

Notice that Theorem 1 is not applicable because if $x=1$ and $y=0.5$, then $d(T(1), S(0.5))=$ 0.9 and

$$
\begin{aligned}
M_{1}(1,0.5)= & \max \{d(A(1), B(0.5)), d(A(1), T(1)), d(B(0.5), S(0.5)), \\
& \frac{d(A(1), S(0.5))+d(T(1), B(0.5))}{2}, \frac{d(A(1), S(0.5)) d(T(1), B(0.5))}{1+d(A(1), B(0.5))}, \\
& \frac{d(A(1), T(1)) d(B(0.5), S(0.5))}{1+d(A(1), B(0.5))}, \\
& \left.\frac{1+d(A(1), S(0.5))+d(T(1), B(0.5))}{1+d(A(1), T(1))+d(B(0.5), S(0.5))} d(A(1), T(1))\right\} \\
= & \max \{0.875,0.1,0.125,0.8875,0.4133,0.00667,0.2265\}=0.8875 .
\end{aligned}
$$

As a result, $d(T(1), S(0))=0.9>0.79875=\psi(0.8875)=\psi\left(M_{1}(1,0.5)\right)$, that is, inequality (1) does not hold.

To be precise, we point out that our contractivity condition holds because we have employed $\phi_{1}(r, s)=\max \left\{r, \frac{r+s}{2}\right\}$, which is better for our kind of contractivity conditions than $\phi_{1}^{\prime}(r, s)=\frac{r+s}{2}$, as Liu et al. used in Theorem 1 .

\section{Common fixed point theorems under $\alpha$ conditions}

The study of fixed point theory in ordered metric spaces was initiated by Ran and Reurings in [36] and by Nieto and Rodríguez-López in [37]. Later, improved results were obtained 
by many authors (see, for instance, $[17,20,22,23,25-27,31,33])$. In this section we obtain a version of Theorem 6 involving a function $\alpha$ that can generalize a partial order.

In the sequel, let $\alpha: X \times X \rightarrow[0, \infty)$ be a function.

Definition 10 We will say that $\alpha$ is reflexive if $\alpha(x, x) \geq 1$ for all $x \in X$.

Definition 11 We will say that $\alpha$ is transitive if $\alpha(x, z) \geq 1$ for all $x, y, z \in X$ such that $\alpha(x, y) \geq 1$ and $\alpha(y, z) \geq 1$.

Definition 12 Given two mappings $f, g: X \rightarrow X$, we will say that $f(X)$ is an $\alpha$-subset of $g(X)$ if for all $x \in X$, there exists $y \in X$ such that $f x=g y$ and $\alpha(x, y) \geq 1$. In such a case, we will write $f(X) \subseteq_{\alpha} g(X)$.

Remark 13 If $f(X) \subseteq_{\alpha} g(X)$ then $f(X) \subseteq g(X)$. If $\alpha(x, y) \geq 1$ for all $x, y \in X$, then $f(X) \subseteq_{\alpha}$ $g(X)$ if, and only if, $f(X) \subseteq g(X)$.

Definition 14 Given two mappings $f, g: X \rightarrow X$, we will say that the pair $\{f, g\}$ is $(\alpha, d)$ regular if

$$
\max \left\{\alpha\left(x_{n}, u\right), \alpha\left(u, x_{n}\right)\right\} \geq 1 \quad \text { for all } n \in \mathbb{N}
$$

provided that $\left\{x_{n}\right\} \subseteq X$ is a sequence such that $\left\{f x_{n}\right\} \rightarrow g u$ and $\alpha\left(x_{n}, x_{n+1}\right) \geq 1$ for all $n \in \mathbb{N}$.

Theorem 15 Let $\alpha: X \times X \rightarrow[0, \infty)$ be a reflexive, transitive function and let $A, B, S, T$ : $X \rightarrow X$ be self-mappings in a metric space $(X, d)$ such that

(a) $\{A, T\}$ and $\{B, S\}$ are weakly compatible;

(b) $T(X) \subseteq_{\alpha} B(X)$ and $S(X) \subseteq_{\alpha} A(X)$;

(c) one of $A(X), B(X), S(X)$, and $T(X)$ is complete;

(d) the pairs $\{T, B\}$ and $\{S, A\}$ are $(\alpha, d)$-regular.

Also assume that there exist $\phi_{1} \in \mathrm{F}_{1}, \phi_{2} \in \mathrm{F}_{2}, \phi_{3} \in \mathrm{F}_{3}, \phi_{4} \in \mathrm{F}_{4}$, and $\psi \in \Phi_{3}$ such that

$$
\max \{\alpha(x, y), \alpha(y, x)\} d(T x, S y) \leq \psi(M(x, y)) \quad \text { for all } x, y \in X
$$

where

$$
\begin{aligned}
M(x, y)= & \max \left\{d(A x, B y), d(A x, T x), d(B y, S y), \phi_{1}(d(A x, S y), d(T x, B y)),\right. \\
& \phi_{2}(d(A x, S y), d(T x, B y), d(A x, B y)), \phi_{3}(d(A x, T x), d(B y, S y), d(A x, B y)) \\
& \left.\phi_{4}(d(A x, S y), d(T x, B y), d(A x, T x), d(B y, S y))\right\} .
\end{aligned}
$$

Then $A, B, S$, and $T$ have a coincidence point in $X$.

Proof Let $x_{0} \in X$ be an arbitrary point and let $y_{1}=T x_{0}$. Since $T x_{0} \in T(X) \subseteq_{\alpha} B(X)$, there exists $x_{1} \in X$ such that $y_{1}=T x_{0}=B x_{1}$ and $\alpha\left(x_{0}, x_{1}\right) \geq 1$. Let $y_{2}=S x_{1}$. Since $S x_{1} \in S(X) \subseteq_{\alpha}$ 
$A(X)$, there exists $x_{2} \in X$ such that $y_{2}=S x_{1}=A x_{2}$ and $\alpha\left(x_{1}, x_{2}\right) \geq 1$. Let $y_{3}=T x_{2}$. Repeating again and again this process, we can find two sequences $\left\{x_{n}\right\}$ and $\left\{y_{n}\right\}$ in $X$ such that, for all $n \in \mathbb{N}_{0}$,

$$
\begin{aligned}
& y_{2 n+1}=B x_{2 n+1}=T x_{2 n}, \quad y_{2 n+2}=A x_{2 n+2}=S x_{2 n+1}, \quad \text { and } \\
& \alpha\left(x_{n}, x_{n+1}\right) \geq 1
\end{aligned}
$$

As $\alpha$ is reflexive and transitive,

$$
\alpha\left(x_{n}, x_{m}\right) \geq 1 \quad \text { for all } n, m \in \mathbb{N} \text { with } n \leq m
$$

Let define $d_{n}=d\left(y_{n}, y_{n+1}\right)$ for all $n \in \mathbb{N}_{0}$. We claim that $\lim _{n \rightarrow \infty} d_{n}=0$. Indeed, by (16) and (18), for all $n \in \mathbb{N}$,

$$
\begin{aligned}
d_{2 n} & =d\left(y_{2 n}, y_{2 n+1}\right)=d\left(y_{2 n+1}, y_{2 n}\right)=d\left(T x_{2 n}, S x_{2 n-1}\right) \\
& \leq \max \left\{\alpha\left(x_{2 n}, x_{2 n-1}\right), \alpha\left(x_{2 n-1}, x_{2 n}\right)\right\} d\left(T x_{2 n}, S x_{2 n-1}\right) \leq \psi\left(M\left(x_{2 n}, x_{2 n-1}\right)\right) .
\end{aligned}
$$

Taking into account the properties that define functions in $\left\{\mathrm{F}_{i}\right\}_{i=1}^{4}$, we deduce that

$$
\begin{aligned}
& d\left(A x_{2 n}, B x_{2 n-1}\right)=d\left(y_{2 n}, y_{2 n-1}\right)=d\left(y_{2 n-1}, y_{2 n}\right)=d_{2 n-1} ; \\
& d\left(A x_{2 n}, T x_{2 n}\right)=d\left(y_{2 n}, y_{2 n+1}\right)=d_{2 n} ; \\
& d\left(B x_{2 n-1}, S x_{2 n-1}\right)=d\left(y_{2 n-1}, y_{2 n}\right)=d_{2 n-1} ; \\
& \phi_{1}\left(d\left(A x_{2 n}, S x_{2 n-1}\right), d\left(T x_{2 n}, B x_{2 n-1}\right)\right) \\
& \quad=\phi_{1}\left(d\left(y_{2 n}, y_{2 n}\right), d\left(y_{2 n+1}, y_{2 n-1}\right)\right)=\phi_{1}\left(0, d\left(y_{2 n-1}, y_{2 n+1}\right)\right) \\
& \quad\left(F_{12}\right) d\left(y_{2 n-1}, y_{2 n+1}\right) \\
& \quad \leq \quad \leq \frac{d\left(y_{2 n-1}, y_{2 n}\right)+d\left(y_{2 n}, y_{2 n+1}\right)}{2} \\
& \quad \leq \max \left\{d\left(y_{2 n+1}, y_{2 n}\right), d\left(y_{2 n}, y_{2 n-1}\right)\right\}=\max \left\{d_{2 n-1}, d_{2 n}\right\} ; \\
& \phi_{2}\left(d\left(A x_{2 n}, S x_{2 n-1}\right), d\left(T x_{2 n}, B x_{2 n-1}\right), d\left(A x_{2 n}, B x_{2 n-1}\right)\right) \\
& \quad=\phi_{2}\left(d\left(y_{2 n}, y_{2 n}\right), d\left(y_{2 n+1}, y_{2 n-1}\right), d\left(y_{2 n}, y_{2 n-1}\right)\right) \\
& \quad=\phi_{2}\left(0, d\left(y_{2 n+1}, y_{2 n-1}\right), d\left(y_{2 n}, y_{2 n-1}\right)\right) \stackrel{\left(F_{22}\right)}{\leq} \max \left\{d_{2 n-1}, d_{2 n}\right\} ; \\
& \quad\left(F_{42}\right) \\
& \quad=\phi_{3}\left(d\left(A x_{2 n}, T x_{2 n}\right), d\left(B x_{2 n-1}, S x_{2 n-1}\right), d\left(A x_{2 n}, B x_{2 n-1}\right)\right) \\
& \quad=\phi_{3}\left(d\left(y_{2 n}, y_{2 n+1}\right), d\left(y_{2 n-1}, y_{2 n}\right), d\left(y_{2 n}, y_{2 n-1}\right)\right) \\
& \quad=\phi_{3}\left(d_{2 n}, d_{2 n-1}, d_{2 n-1}\right) \stackrel{\left(F_{32}\right)}{\leq} \max \left\{d_{2 n-1}, d_{2 n}\right\} ; \\
& \phi_{4}\left(d\left(A x_{2 n}, S x_{2 n-1}\right), d\left(T x_{2 n}, B x_{2 n-1}\right), d\left(A x_{2 n}, T x_{2 n}\right), d\left(B x_{2 n-1}, S x_{2 n-1}\right)\right) \\
& \left.\left.\quad 2, y_{2 n}\right), d\left(y_{2 n+1}, y_{2 n-1}\right), d\left(y_{2 n}, y_{2 n+1}\right), d\left(y_{2 n-1}, y_{2 n}\right)\right) \\
& \quad \\
& \quad
\end{aligned}
$$




$$
\begin{aligned}
& \leq \max \left\{\frac{d\left(y_{2 n-1}, y_{2 n}\right)+d\left(y_{2 n}, y_{2 n+1}\right)}{2}, d\left(y_{2 n}, y_{2 n+1}\right), d\left(y_{2 n-1}, y_{2 n}\right)\right\} \\
& =\max \left\{d_{2 n-1}, d_{2 n}\right\} .
\end{aligned}
$$

Hence,

$$
\begin{aligned}
M\left(x_{2 n}, x_{2 n-1}\right)= & \max \left\{d\left(A x_{2 n}, B x_{2 n-1}\right), d\left(A x_{2 n}, T x_{2 n}\right), d\left(B x_{2 n-1}, S x_{2 n-1}\right),\right. \\
& \phi_{1}\left(d\left(A x_{2 n}, S x_{2 n-1}\right), d\left(T x_{2 n}, B x_{2 n-1}\right)\right), \\
& \phi_{2}\left(d\left(A x_{2 n}, S x_{2 n-1}\right), d\left(T x_{2 n}, B x_{2 n-1}\right), d\left(A x_{2 n}, B x_{2 n-1}\right)\right), \\
& \phi_{3}\left(d\left(A x_{2 n}, T x_{2 n}\right), d\left(B x_{2 n-1}, S x_{2 n-1}\right), d\left(A x_{2 n}, B x_{2 n-1}\right)\right), \\
& \left.\phi_{4}\left(d\left(A x_{2 n}, S x_{2 n-1}\right), d\left(T x_{2 n}, B x_{2 n-1}\right), d\left(A x_{2 n}, T x_{2 n}\right), d\left(B x_{2 n-1}, S x_{2 n-1}\right)\right)\right\} \\
= & \max \left\{d_{2 n-1}, d_{2 n}\right\} .
\end{aligned}
$$

By (19),

$$
d_{2 n} \leq \psi\left(M\left(x_{2 n}, x_{2 n-1}\right)\right)=\psi\left(\max \left\{d_{2 n-1}, d_{2 n}\right\}\right) .
$$

If $d_{2 n-1}<d_{2 n}$ for some $n$, then $d_{2 n}>0$ and we get the contradiction $d_{2 n} \leq \psi\left(\max \left\{d_{2 n-1}\right.\right.$, $\left.\left.d_{2 n}\right\}\right)=\psi\left(d_{2 n}\right)$ (recall Lemma 4). As a consequence, we deduce that $d_{2 n} \leq d_{2 n-1}$ for all $n \in \mathbb{N}$. Similarly, it can be proved that $d_{2 n+1} \leq \psi\left(\left\{d_{2 n}, d_{2 n+1}\right\}\right)$, so $d_{2 n+1} \leq d_{2 n}$ for all $n \in \mathbb{N}$. Hence,

$$
d_{n+1} \leq \psi\left(d_{n}\right) \quad \text { and } \quad d_{n+1} \leq d_{n} \quad \text { for all } n \in \mathbb{N} \text {. }
$$

Since $\left\{d_{n}\right\}$ is a non-increasing sequence of non-negative real numbers, it is convergent. Let $L \geq 0$ be its limit. Taking into account that $\psi \in \Phi_{3}$ and $d_{n+1} \leq \psi\left(d_{n}\right)$ for all $n \in \mathbb{N}$, we deduce that $\left\{d_{n}\right\} \rightarrow 0$, that is,

$$
\lim _{n \rightarrow \infty} d\left(y_{n}, y_{n+1}\right)=0
$$

Next, let us show that $\left\{y_{n}\right\}$ is a Cauchy sequence in $(X, d)$ reasoning by contradiction. Assume that $\left\{y_{n}\right\}$ is not a Cauchy sequence. By Proposition 5, the sequence $\left\{y_{2 n}\right\}$ is not a Cauchy sequence. In particular, there exist $\varepsilon_{0}>0$ and two subsequences $\left\{y_{2 n(k)}\right\}$ and $\left\{y_{2 m(k)}\right\}$ of $\left\{y_{2 n}\right\}$ such that

$$
\begin{aligned}
2 k<2 n(k)<2 m(k), & d\left(y_{2 n(k)}, y_{2 m(k)-2}\right) \leq \varepsilon_{0}<d\left(y_{2 n(k)}, y_{2 m(k)}\right) \quad \text { for all } k \in \mathbb{N}, \\
\lim _{n \rightarrow \infty} d\left(y_{2 n(k)}, y_{2 m(k)}\right)= & \lim _{n \rightarrow \infty} d\left(y_{2 n(k)}, y_{2 m(k)-1}\right)=\lim _{n \rightarrow \infty} d\left(y_{2 n(k)}, y_{2 m(k)+1}\right) \\
= & \lim _{n \rightarrow \infty} d\left(y_{2 n(k)-1}, y_{2 m(k)-1}\right)=\lim _{n \rightarrow \infty} d\left(y_{2 n(k)-1}, y_{2 m(k)+1}\right)=\varepsilon_{0} .
\end{aligned}
$$

By (16) and (18), for all $k \in \mathbb{N}$,

$$
\begin{aligned}
d\left(y_{2 m(k)+1}, y_{2 n(k)}\right) & =d\left(T x_{2 m(k)}, S x_{2 n(k)-1}\right) \\
& \leq \max \left\{\alpha\left(x_{2 m(k)}, x_{2 n(k)-1}\right), \alpha\left(x_{2 n(k)-1}, x_{2 m(k)}\right)\right\} d\left(T x_{2 m(k)}, S x_{2 n(k)-1}\right) \\
& \leq \psi\left(M\left(x_{2 m(k)}, x_{2 n(k)-1}\right)\right) .
\end{aligned}
$$


Notice that

$$
\begin{aligned}
& \lim _{k \rightarrow \infty} d\left(A x_{2 m(k)}, B x_{2 n(k)-1}\right)=\lim _{k \rightarrow \infty} d\left(y_{2 m(k)}, y_{2 n(k)-1}\right)=\varepsilon_{0} ; \\
& \lim _{k \rightarrow \infty} d\left(A x_{2 m(k)}, T x_{2 m(k)}\right)=\lim _{k \rightarrow \infty} d\left(y_{2 m(k)}, y_{2 m(k)+1}\right)=0 ; \\
& \lim _{k \rightarrow \infty} d\left(B x_{2 n(k)-1}, S x_{2 n(k)-1}\right)=\lim _{k \rightarrow \infty} d\left(y_{2 n(k)-1}, y_{2 n(k)}\right)=0 ; \\
& \lim _{k \rightarrow \infty} \phi_{1}\left(d\left(A x_{2 m(k)}, S x_{2 n(k)-1}\right), d\left(T x_{2 m(k)}, B x_{2 n(k)-1}\right)\right) \\
& =\lim _{k \rightarrow \infty} \phi_{1}\left(d\left(y_{2 m(k)}, y_{2 n(k)}\right), d\left(y_{2 m(k)+1}, y_{2 n(k)-1}\right)\right) \\
& \stackrel{\left(F_{11}\right)}{=} \phi_{1}\left(\lim _{k \rightarrow \infty} d\left(y_{2 m(k)}, y_{2 n(k)}\right), \lim _{k \rightarrow \infty} d\left(y_{2 m(k)+1}, y_{2 n(k)-1}\right)\right)=\phi_{1}\left(\varepsilon_{0}, \varepsilon_{0}\right) \stackrel{\left(F_{13}\right)}{\leq} \varepsilon_{0} ; \\
& \lim _{k \rightarrow \infty} \phi_{2}\left(d\left(A x_{2 m(k)}, S x_{2 n(k)-1}\right), d\left(T x_{2 m(k)}, B x_{2 n(k)-1}\right), d\left(A x_{2 m(k)}, B x_{2 n(k)-1}\right)\right) \\
& =\lim _{k \rightarrow \infty} \phi_{2}\left(d\left(y_{2 m(k)}, y_{2 n(k)}\right), d\left(y_{2 m(k)+1}, y_{2 n(k)-1}\right), d\left(y_{2 m(k)}, y_{2 n(k)-1}\right)\right) \\
& \stackrel{\left(F_{21}\right)}{=} \phi_{2}\left(\lim _{k \rightarrow \infty} d\left(y_{2 m(k)}, y_{2 n(k)}\right), \lim _{k \rightarrow \infty} d\left(y_{2 m(k)+1}, y_{2 n(k)-1}\right), \lim _{k \rightarrow \infty} d\left(y_{2 m(k)}, y_{2 n(k)-1}\right)\right) \\
& =\phi_{2}\left(\varepsilon_{0}, \varepsilon_{0}, \varepsilon_{0}\right) \stackrel{\left(\mathrm{F}_{23}\right)}{\leq} \varepsilon_{0} \\
& \lim _{k \rightarrow \infty} \phi_{3}\left(d\left(A x_{2 m(k)}, T x_{2 m(k)}\right), d\left(B x_{2 n(k)-1}, S x_{2 n(k)-1}\right), d\left(A x_{2 m(k)}, B x_{2 n(k)-1}\right)\right) \\
& =\lim _{k \rightarrow \infty} \phi_{3}\left(d\left(y_{2 m(k)}, y_{2 m(k)+1}\right), d\left(y_{2 n(k)-1}, y_{2 n(k)}\right), d\left(y_{2 m(k)}, y_{2 n(k)-1}\right)\right) \\
& \stackrel{\left(\mathrm{F}_{31}\right)}{=} \phi_{3}\left(\lim _{k \rightarrow \infty} d\left(y_{2 m(k)}, y_{2 m(k)+1}\right), \lim _{k \rightarrow \infty} d\left(y_{2 n(k)-1}, y_{2 n(k)}\right), \lim _{k \rightarrow \infty} d\left(y_{2 m(k)}, y_{2 n(k)-1}\right)\right) \\
& =\phi_{3}\left(0,0, \varepsilon_{0}\right) \stackrel{\left(F_{33}\right)}{\leq} \varepsilon_{0} \\
& \lim _{k \rightarrow \infty} \phi_{4}\left(d\left(A x_{2 m(k)}, S x_{2 n(k)-1}\right), d\left(T x_{2 m(k)}, B x_{2 n(k)-1}\right), d\left(A x_{2 m(k)}, T x_{2 m(k)}\right),\right. \\
& \left.d\left(B x_{2 n(k)-1}, S x_{2 n(k)-1}\right)\right) \\
& =\lim _{k \rightarrow \infty} \phi_{4}\left(d\left(y_{2 m(k)}, y_{2 n(k)}\right), d\left(y_{2 m(k)+1}, y_{2 n(k)-1}\right), d\left(y_{2 m(k)}, y_{2 m(k)+1}\right), d\left(y_{2 n(k)-1}, y_{2 n(k)}\right)\right) \\
& \stackrel{\left(F_{41}\right)}{=} \phi_{4}\left(\lim _{k \rightarrow \infty} d\left(y_{2 m(k)}, y_{2 n(k)}\right), \lim _{k \rightarrow \infty} d\left(y_{2 m(k)+1}, y_{2 n(k)-1}\right)\right. \text {, } \\
& \left.\lim _{k \rightarrow \infty} d\left(y_{2 m(k)}, y_{2 m(k)+1}\right), \lim _{k \rightarrow \infty} d\left(y_{2 n(k)-1}, y_{2 n(k)}\right)\right) \\
& =\phi_{4}\left(\varepsilon_{0}, \varepsilon_{0}, 0,0\right) \stackrel{\left(\mathrm{F}_{43}\right)}{\leq} \varepsilon_{0} \text {. }
\end{aligned}
$$

As a result, it follows from (21) and (20) that

$$
\begin{aligned}
\lim _{k \rightarrow \infty} M\left(x_{2 m(k)}, x_{2 n(k)-1}\right) \\
=\lim _{k \rightarrow \infty} \max \left\{d\left(A x_{2 m(k)}, B x_{2 n(k)-1}\right), d\left(A x_{2 m(k)}, T x_{2 m(k)}\right), d\left(B x_{2 n(k)-1}, S x_{2 n(k)-1}\right),\right. \\
\quad \phi_{1}\left(d\left(A x_{2 m(k)}, S x_{2 n(k)-1}\right), d\left(T x_{2 m(k)}, B x_{2 n(k)-1}\right)\right), \\
\quad \phi_{2}\left(d\left(A x_{2 m(k)}, S x_{2 n(k)-1}\right), d\left(T x_{2 m(k)}, B x_{2 n(k)-1}\right), d\left(A x_{2 m(k)}, B x_{2 n(k)-1}\right)\right),
\end{aligned}
$$




$$
\begin{aligned}
& \phi_{3}\left(d\left(A x_{2 m(k)}, T x_{2 m(k)}\right), d\left(B x_{2 n(k)-1}, S x_{2 n(k)-1}\right), d\left(A x_{2 m(k)}, B x_{2 n(k)-1}\right)\right), \\
& \phi_{4}\left(d\left(A x_{2 m(k)}, S x_{2 n(k)-1}\right), d\left(T x_{2 m(k)}, B x_{2 n(k)-1}\right), d\left(A x_{2 m(k)}, T x_{2 m(k)}\right),\right. \\
& \left.\left.d\left(B x_{2 n(k)-1}, S x_{2 n(k)-1}\right)\right)\right\} \\
= & \varepsilon_{0} .
\end{aligned}
$$

As $\psi$ is upper semi-continuous, we deduce that

$$
\begin{aligned}
\varepsilon_{0} & =\lim _{n \rightarrow \infty} d\left(y_{2 m(k)+1}, y_{2 n(k)}\right)=\limsup _{n \rightarrow \infty} d\left(y_{2 m(k)+1}, y_{2 n(k)}\right) \\
& \leq \limsup _{n \rightarrow \infty} \psi\left(M\left(x_{2 m(k)}, x_{2 n(k)-1}\right)\right) \leq \psi\left(\varepsilon_{0}\right)<\varepsilon_{0},
\end{aligned}
$$

which is a contradiction. As a result, $\left\{y_{n}\right\}$ is a Cauchy sequence in $(X, d)$. Then, there exists $z \in X$ such that $\left\{y_{n}\right\} \rightarrow z$. In particular,

$$
z=\lim _{n \rightarrow \infty} y_{n}=\lim _{n \rightarrow \infty} T x_{2 n}=\lim _{n \rightarrow \infty} B x_{2 n+1}=\lim _{n \rightarrow \infty} A x_{2 n}=\lim _{n \rightarrow \infty} S x_{2 n-1} .
$$

Next we distinguish some cases depending on the complete set. Notice that

$$
\begin{aligned}
& \left\{y_{2 n+2}=A x_{2 n+2}=S x_{2 n+1}\right\} \subseteq S(X) \subseteq A(X) \text { and } \\
& \left\{y_{2 n+1}=B x_{2 n+1}=T x_{2 n}\right\} \subseteq T(X) \subseteq B(X)
\end{aligned}
$$

Assume that $A(X)$ (or $S(X)$ ) is complete. Then there exists $u \in X$ such that $z=A u$. To show that $T u=z$, suppose, by contradiction, that $d(T u, z)>0$. As the pair $\{S, A\}$ is $(\alpha, d)$-regular and $\left\{y_{2 n+2}=S x_{2 n+1}\right\} \rightarrow z=A u$, we have

$$
\max \left\{\alpha\left(u, x_{2 n+1}\right), \alpha\left(u, x_{2 n+1}\right)\right\} \geq 1 \quad \text { for all } n \in \mathbb{N}_{0} .
$$

Therefore, for all $n \in \mathbb{N}$,

$$
\begin{aligned}
d\left(T u, y_{2 n+2}\right) & =d\left(T u, S x_{2 n+1}\right) \leq \max \left\{\alpha\left(u, x_{2 n+1}\right), \alpha\left(u, x_{2 n+1}\right)\right\} d\left(T u, S x_{2 n+1}\right) \\
& \leq \psi\left(M\left(u, x_{2 n+1}\right)\right) .
\end{aligned}
$$

Notice that

$$
\begin{aligned}
& \lim _{n \rightarrow \infty} d\left(A u, B x_{2 n+1}\right)=\lim _{n \rightarrow \infty} d\left(z, y_{2 n+1}\right)=0 ; \\
& \lim _{n \rightarrow \infty} d(A u, T u)=d(z, T u)>0 ; \\
& \lim _{n \rightarrow \infty} d\left(B x_{2 n+1}, S x_{2 n+1}\right)=d\left(y_{2 n+1}, y_{2 n+2}\right)=0 ; \\
& \lim _{n \rightarrow \infty} \phi_{1}\left(d\left(A u, S x_{2 n+1}\right), d\left(T u, B x_{2 n+1}\right)\right) \\
& =\lim _{n \rightarrow \infty} \phi_{1}\left(d\left(z, y_{2 n+2}\right), d\left(T u, y_{2 n+1}\right)\right) \\
& \stackrel{\left(F_{11}\right)}{=} \phi_{1}\left(\lim _{n \rightarrow \infty} d\left(z, y_{2 n+2}\right), \lim _{n \rightarrow \infty} d\left(T u, y_{2 n+1}\right)\right) \\
& =\phi_{1}(0, d(T u, z)) \stackrel{\left(F_{12}\right)}{\leq} \frac{d(T u, z)}{2}<d(T u, z) ;
\end{aligned}
$$




$$
\begin{aligned}
& \lim _{n \rightarrow \infty} \phi_{2}\left(d\left(A u, S x_{2 n+1}\right), d\left(T u, B x_{2 n+1}\right), d\left(A u, B x_{2 n+1}\right)\right) \\
& =\lim _{n \rightarrow \infty} \phi_{2}\left(d\left(z, y_{2 n+2}\right), d\left(T u, y_{2 n+1}\right), d\left(z, y_{2 n+1}\right)\right) \\
& \stackrel{\left(\mathrm{F}_{21}\right)}{=} \phi_{2}\left(\lim _{n \rightarrow \infty} d\left(z, y_{2 n+2}\right), \lim _{n \rightarrow \infty} d\left(T u, y_{2 n+1}\right), \lim _{n \rightarrow \infty} d\left(z, y_{2 n+1}\right)\right) \\
& =\phi_{2}(0, d(T u, z), 0) \stackrel{\left(F_{22}\right)}{\leq} d(T u, z) \text {; } \\
& \lim _{n \rightarrow \infty} \phi_{3}\left(d(A u, T u), d\left(B x_{2 n+1}, S x_{2 n+1}\right), d\left(A u, B x_{2 n+1}\right)\right) \\
& =\lim _{n \rightarrow \infty} \phi_{3}\left(d(z, T u), d\left(y_{2 n+1}, y_{2 n+2}\right), d\left(z, y_{2 n+1}\right)\right) \\
& \stackrel{\left(\mathrm{F}_{31}\right)}{=} \phi_{3}\left(\lim _{n \rightarrow \infty} d(z, T u), \lim _{n \rightarrow \infty} d\left(y_{2 n+1}, y_{2 n+2}\right), \lim _{n \rightarrow \infty} d\left(z, y_{2 n+1}\right)\right) \\
& =\phi_{3}(d(z, T u), 0,0) \stackrel{\left(F_{32}\right)}{\leq} d(z, T u) \text {; } \\
& \lim _{n \rightarrow \infty} \phi_{4}\left(d\left(A u, S x_{2 n+1}\right), d\left(T u, B x_{2 n+1}\right), d(A u, T u), d\left(B x_{2 n+1}, S x_{2 n+1}\right)\right) \\
& =\lim _{n \rightarrow \infty} \phi_{4}\left(d\left(z, y_{2 n+2}\right), d\left(T u, y_{2 n+1}\right), d(z, T u), d\left(y_{2 n+1}, y_{2 n+2}\right)\right) \\
& \stackrel{\left(\mathrm{F}_{41}\right)}{=} \phi_{4}\left(\lim _{n \rightarrow \infty} d\left(z, y_{2 n+2}\right), \lim _{n \rightarrow \infty} d\left(T u, y_{2 n+1}\right), \lim _{n \rightarrow \infty} d(z, T u), \lim _{n \rightarrow \infty} d\left(y_{2 n+1}, y_{2 n+2}\right)\right) \\
& =\phi_{4}(0, d(T u, z), d(z, T u), 0) \stackrel{\left(F_{42}\right)}{\leq} d(z, T u) \text {. }
\end{aligned}
$$

Since

$$
\begin{aligned}
& \lim _{n \rightarrow \infty} M\left(u, x_{2 n+1}\right) \\
&=\lim _{n \rightarrow \infty} \max \left\{d\left(A u, B x_{2 n+1}\right), d(A u, T u), d\left(B x_{2 n+1}, S x_{2 n+1}\right),\right. \\
& \quad \phi_{1}\left(d\left(A u, S x_{2 n+1}\right), d\left(T u, B x_{2 n+1}\right)\right), \\
& \quad \phi_{2}\left(d\left(A u, S x_{2 n+1}\right), d\left(T u, B x_{2 n+1}\right), d\left(A u, B x_{2 n+1}\right)\right), \\
& \phi_{3}\left(d(A u, T u), d\left(B x_{2 n+1}, S x_{2 n+1}\right), d\left(A u, B x_{2 n+1}\right)\right), \\
&\left.\phi_{4}\left(d\left(A u, S x_{2 n+1}\right), d\left(T u, B x_{2 n+1}\right), d(A u, T u), d\left(B x_{2 n+1}, S x_{2 n+1}\right)\right)\right\} \\
&= d(z, T u)>0,
\end{aligned}
$$

letting $n \rightarrow \infty$ in (22), the upper semi-continuity of $\psi$ yields

$$
\begin{aligned}
d(z, T u) & =\limsup _{n \rightarrow \infty} d\left(T u, y_{2 n+2}\right) \leq \limsup _{n \rightarrow \infty} \psi\left(M\left(u, x_{2 n+1}\right)\right) \\
& \leq \psi\left(\limsup _{n \rightarrow \infty} M\left(u, x_{2 n+1}\right)\right)=\psi(d(z, T u))<d(z, T u),
\end{aligned}
$$

which is a contradiction. As a consequence, $T u=z=A u$. As $T(X) \subseteq_{\alpha} B(X)$, there exists $w \in X$ such that $T u=B w$ and $\alpha(u, w) \geq 1$. Then $A u=T u=z=B w$. We claim that $z=S w$. To prove it, suppose that $d(z, S w)>0$. As the pair $\{T, B\}$ is $(\alpha, d)$-regular and $\left\{y_{2 n+1}=T x_{2 n}\right\} \rightarrow$ $z=B w$,

$$
\max \left\{\alpha\left(x_{2 n}, w\right), \alpha\left(w, x_{2 n}\right)\right\} \geq 1 \quad \text { for all } n \in \mathbb{N}_{0}
$$


By (16), for all $n \in \mathbb{N}$,

$$
\begin{aligned}
d\left(y_{2 n+1}, S w\right) & =d\left(T x_{2 n}, S w\right) \leq \max \left\{\alpha\left(x_{2 n}, w\right), \alpha\left(w, x_{2 n}\right)\right\} d\left(T x_{2 n}, S w\right) \\
& \leq \psi\left(M\left(x_{2 n}, w\right)\right) .
\end{aligned}
$$

Notice that

$$
\begin{aligned}
& \lim _{n \rightarrow \infty} d\left(A x_{2 n}, B w\right)=\lim _{n \rightarrow \infty} d\left(y_{2 n}, z\right)=0 ; \\
& \lim _{n \rightarrow \infty} d\left(A x_{2 n}, T x_{2 n}\right)=\lim _{n \rightarrow \infty} d\left(y_{2 n}, y_{2 n+1}\right)=0 ; \\
& \lim _{n \rightarrow \infty} d(B w, S w)=d(z, S w)>0 ; \\
& \lim _{n \rightarrow \infty} \phi_{1}\left(d\left(A x_{2 n}, S w\right), d\left(T x_{2 n}, B w\right)\right) \\
& =\lim _{n \rightarrow \infty} \phi_{1}\left(d\left(y_{2 n}, S w\right), d\left(y_{2 n+1}, z\right)\right) \\
& \stackrel{\left(F_{11}\right)}{=} \phi_{1}\left(\lim _{n \rightarrow \infty} d\left(y_{2 n}, S w\right), \lim _{n \rightarrow \infty} d\left(y_{2 n+1}, z\right)\right)=\phi_{1}(d(z, S w), 0) \stackrel{\left(F_{14}\right)}{\leq} d(z, S w) ; \\
& \lim _{n \rightarrow \infty} \phi_{2}\left(d\left(A x_{2 n}, S w\right), d\left(T x_{2 n}, B w\right), d\left(A x_{2 n}, B w\right)\right) \\
& =\lim _{n \rightarrow \infty} \phi_{2}\left(d\left(y_{2 n}, S w\right), d\left(y_{2 n+1}, z\right), d\left(y_{2 n}, z\right)\right) \\
& \stackrel{\left(F_{21}\right)}{=} \phi_{2}\left(\lim _{n \rightarrow \infty} d\left(y_{2 n}, S w\right), \lim _{n \rightarrow \infty} d\left(y_{2 n+1}, z\right), \lim _{n \rightarrow \infty} d\left(y_{2 n}, z\right)\right) \\
& =\phi_{2}(d(z, S w), 0,0) \stackrel{\left(F_{24}\right)}{\leq} d(z, S w) ; \\
& \lim _{n \rightarrow \infty} \phi_{3}\left(d\left(A x_{2 n}, T x_{2 n}\right), d(B w, S w), d\left(A x_{2 n}, B w\right)\right) \\
& =\lim _{n \rightarrow \infty} \phi_{3}\left(d\left(y_{2 n}, y_{2 n+1}\right), d(z, S w), d\left(y_{2 n}, z\right)\right) \\
& \stackrel{\left(F_{31}\right)}{=} \phi_{3}\left(\lim _{n \rightarrow \infty} d\left(y_{2 n}, y_{2 n+1}\right), \lim _{n \rightarrow \infty} d(z, S w), \lim _{n \rightarrow \infty} d\left(y_{2 n}, z\right)\right) \\
& =\phi_{3}(0, d(z, S w), 0) \stackrel{\left(F_{34}\right)}{\leq} d(z, S w) ; \\
& \lim _{n \rightarrow \infty} \phi_{4}\left(d\left(A x_{2 n}, S w\right), d\left(T x_{2 n}, B w\right), d\left(A x_{2 n}, T x_{2 n}\right), d(B w, S w)\right) \\
& =\lim _{n \rightarrow \infty} \phi_{4}\left(d\left(y_{2 n}, S w\right), d\left(y_{2 n+1}, z\right), d\left(y_{2 n}, y_{2 n+1}\right), d(z, S w)\right) \\
& \stackrel{\left(F_{41}\right)}{=} \phi_{4}\left(\lim _{n \rightarrow \infty} d\left(y_{2 n}, S w\right), \lim _{n \rightarrow \infty} d\left(y_{2 n+1}, z\right), \lim _{n \rightarrow \infty} d\left(y_{2 n}, y_{2 n+1}\right), \lim _{n \rightarrow \infty} d(z, S w)\right) \\
& =\phi_{4}(d(z, S w), 0,0, d(z, S w)) \stackrel{\left(F_{44}\right)}{\leq} d(z, S w) \text {. }
\end{aligned}
$$

Hence,

$$
\begin{aligned}
\lim _{n \rightarrow \infty} M\left(x_{2 n}, w\right)= & \lim _{n \rightarrow \infty} \max \left\{d\left(A x_{2 n}, B w\right), d\left(A x_{2 n}, T x_{2 n}\right), d(B w, S w),\right. \\
& \phi_{1}\left(d\left(A x_{2 n}, S w\right), d\left(T x_{2 n}, B w\right)\right), \\
& \phi_{2}\left(d\left(A x_{2 n}, S w\right), d\left(T x_{2 n}, B w\right), d\left(A x_{2 n}, B w\right)\right) \\
& \phi_{3}\left(d\left(A x_{2 n}, T x_{2 n}\right), d(B w, S w), d\left(A x_{2 n}, B w\right)\right)
\end{aligned}
$$




$$
\begin{aligned}
& \left.\phi_{4}\left(d\left(A x_{2 n}, S w\right), d\left(T x_{2 n}, B w\right), d\left(A x_{2 n}, T x_{2 n}\right), d(B w, S w)\right)\right\} \\
= & d(z, S w)>0 .
\end{aligned}
$$

Again, letting $n \rightarrow \infty$ in (23) and using the upper semi-continuity of $\psi$, we deduce that

$$
\begin{aligned}
d(z, S w) & =\limsup _{n \rightarrow \infty} d\left(y_{2 n+1}, S w\right) \leq \limsup _{n \rightarrow \infty} \psi\left(M\left(x_{2 n}, w\right)\right) \\
& \leq \psi\left(\lim _{n \rightarrow \infty} M\left(x_{2 n}, w\right)\right)=\psi(d(z, S w))<d(z, S w)
\end{aligned}
$$

which is a contradiction. Thus, $S w=z$, which means that $z=T u=A u=B w=S w$. As $\{A, T\}$ and $\{B, S\}$ are weakly compatible,

$$
A z=A T u=T A u=T z \quad \text { and } \quad B z=B S w=S B w=S z .
$$

Next, let us show that $T z=S z$. On the contrary case, suppose that $d(T z, S z)>0$. Hence, by (16),

$$
d(T z, S z) \leq \max \{\alpha(z, z), \alpha(z, z)\} d(T z, S z) \leq \psi(M(z, z)) .
$$

Notice that

$$
\begin{aligned}
& d(A z, B z)=d(T z, S z)>0 ; \\
& d(A z, T z)=d(T z, T z)=0 ; \\
& d(B z, S z)=d(S z, S z)=0 ; \\
& \phi_{1}(d(A z, S z), d(T z, B z))=\phi_{1}(d(T z, S z), d(T z, S z)) \stackrel{\left(F_{13}\right)}{\leq} d(T z, S z) ; \\
& \phi_{2}(d(A z, S z), d(T z, B z), d(A z, B z))=\phi_{2}(d(T z, S z), d(T z, S z), d(T z, S z)) \stackrel{\left(F_{23}\right)}{\leq} d(T z, S z) ; \\
& \phi_{3}(d(A z, T z), d(B z, S z), d(A z, B z))=\phi_{3}(d(T z, T z), d(S z, S z), d(T z, S z)) \\
& \quad=\phi_{3}(0,0, d(T z, S z)) \stackrel{\left(F_{33}\right)}{\leq} d(T z, S z) ; \\
& \quad=\phi_{4}(d(A z, S z), d(T z, B z), d(A z, T z), d(B z, S z)) \\
& \quad=\phi_{4}(d(T z, S z), d(T z, S z), 0,0) \stackrel{\left(F_{43}\right)}{\leq} d(T z, S z) .
\end{aligned}
$$

Therefore,

$$
\begin{aligned}
M(z, z)= & \max \{d(A z, B z), d(A z, T z), d(B z, S z) \\
& \phi_{1}(d(A z, S z), d(T z, B z)), \phi_{2}(d(A z, S z), d(T z, B z), d(A z, B z)) \\
& \phi_{3}(d(A z, T z), d(B z, S z), d(A z, B z)) \\
& \left.\phi_{4}(d(A z, S z), d(T z, B z), d(A z, T z), d(B z, S z))\right\} \\
= & d(T z, S z)>0
\end{aligned}
$$


Again, it follows from (24) that

$$
d(T z, S z) \leq \psi(M(z, z))=\psi(d(T z, S z))<d(T z, S z)
$$

which is a contradiction. As a consequence, we conclude that $T z=S z$, which means that

$$
A z=T z=S z=B z .
$$

In particular, $z$ is a coincidence point of $A, B, T$, and $S$.

In the following result, we describe some sufficient conditions to ensure that the coincidence point is a common fixed point, and it is unique.

Theorem 16 Under the hypotheses of Theorem 15, suppose that $\max \{\alpha(z, w), \alpha(w, z)\} \geq 1$ for all coincidence point $z$ of $A, B, S$, and $T$, and all $w \in S^{-1}(\{z\})$. Then $A, B, S$, and $T$ have, at least, a common fixed point.

Furthermore, if we additionally assume that $\max \{\alpha(x, y), \alpha(y, x)\} \geq 1$ for all distinct common fixed points $x$ and $y$ of $A, B, S$, and $T$, then $A, B, S$, and $T$ have a unique common fixed point.

Proof In such a case, we can repeat, point by point, the arguments of the proof of Theorem 6 in order to demonstrate the following facts:

- $T z=z$ as in (13), so $z$ is a common fixed point of $A, B, S$, and $T$;

- $z$ is the unique common fixed point of $A, B, S$, and $T$, as in (14).

This completes the proof.

The following result corresponds to the case in which $T=B$ and $S=A$ in Theorem 15 .

Corollary 17 Let $\alpha: X \times X \rightarrow[0, \infty)$ be a reflexive, transitive function and let $T, S: X \rightarrow X$ be self-mappings in a metric space $(X, d)$ such that

(a) the pair $\{T, S\}$ is weakly compatible;

(b) one of $T(X)$ or $S(X)$ is complete.

Also assume that there exist $\phi_{1} \in \mathrm{F}_{1}, \phi_{2} \in \mathrm{F}_{2}, \phi_{3} \in \mathrm{F}_{3}, \phi_{4} \in \mathrm{F}_{4}$, and $\psi \in \Phi_{3}$ such that

$$
\max \{\alpha(x, y), \alpha(y, x)\} d(T x, S y) \leq \psi(M(x, y)) \quad \text { for all } x, y \in X
$$

where

$$
\begin{aligned}
M(x, y)= & \max \left\{d(S x, T y), d(S x, T x), d(T y, S y), \phi_{1}(d(S x, S y), d(T x, T y)),\right. \\
& \phi_{2}(d(S x, S y), d(T x, T y), d(S x, T y)), \phi_{3}(d(S x, T x), d(T y, S y), d(S x, T y)) \\
& \left.\phi_{4}(d(S x, S y), d(T x, T y), d(S x, T x), d(T y, S y))\right\} .
\end{aligned}
$$

Then $T$ and S have a coincidence point in $X$. 
Remark 18 Theorem 15 can be particularized to the case in which the metric space $(X, d)$ is endowed with a partial order $\preccurlyeq$ (or even with a reflexive, transitive binary relation) by defining

$$
\alpha_{\preccurlyeq}(x, y)= \begin{cases}1, & \text { if } x \preccurlyeq y, \\ 0, & \text { otherwise. }\end{cases}
$$

In such a case, the contractivity condition (16) turns into the following one:

$$
d(T x, S y) \leq \psi(M(x, y)) \quad \text { for all } x, y \in X \text { such that } x \preccurlyeq y .
$$

In [38-40], the authors illustrated their main results with a list of several corollaries by choosing the function $\alpha$ in different ways. We could repeat here their studies but, for short, we only describe the case in which $S=T$, and $A$ and $B$ are the identity mapping $I_{X}$ in $X$ in Theorem 15.

Corollary 19 Let $\alpha: X \times X \rightarrow[0, \infty)$ be a reflexive, transitive function and let $T: X \rightarrow X$ be a self-mapping in a metric space $(X, d)$ such that

(b) $\alpha(x, T x) \geq 1$ for all $x \in X$;

(c) $X($ or $T(X))$ is complete;

(d) the pair $\left\{T, I_{X}\right\}$ is $(\alpha, d)$-regular.

Also assume that there exist $\phi_{1} \in \mathrm{F}_{1}, \phi_{2} \in \mathrm{F}_{2}, \phi_{3} \in \mathrm{F}_{3}, \phi_{4} \in \mathrm{F}_{4}$, and $\psi \in \Phi_{3}$ such that

$$
\max \{\alpha(x, y), \alpha(y, x)\} d(T x, T y) \leq \psi(M(x, y)) \quad \text { for all } x, y \in X,
$$

where

$$
\begin{aligned}
M(x, y)= & \max \left\{d(x, y), d(x, T x), d(y, T y), \phi_{1}(d(x, T y), d(T x, y)),\right. \\
& \phi_{2}(d(x, T y), d(T x, y), d(x, y)), \phi_{3}(d(x, T x), d(y, T y), d(x, y)) \\
& \left.\phi_{4}(d(x, T y), d(T x, y), d(x, T x), d(y, T y))\right\} .
\end{aligned}
$$

Then $T$ has, at least, a fixed point in $X$.

Competing interests

The authors declare that they have no competing interests.

Authors' contributions

All authors contributed equally and significantly in writing this article. All authors read and approved the final manuscript.

\section{Author details}

'Department of Mathematics, Texas A\&M University-Kingsville, Kingsville, TX 78363, USA. ²Department of Mathematics, Faculty of Science, King Abdulaziz University, Jeddah, 21589, Saudi Arabia. ${ }^{3}$ Nonlinear Analysis and Applied Mathematics Research Group (NAAM), King Abdulaziz University, Jeddah, Saudi Arabia. ${ }^{4}$ Department of Mathematics, Atilim University, Incek, Ankara, 06836, Turkey. ${ }^{5}$ Department of Mathematics, University of Jaén, Campus las Lagunillas s/n, Jaén, 23071, Spain.

Received: 7 May 2015 Accepted: 15 June 2015 Published online: 02 July 2015 


\section{References}

1. Banach, S: Sur les opérations dans les ensembles abstraits et leur application aux équations intégrales. Fundam. Math. 3, 133-181 (1922)

2. Rhoades, BE: A comparison of various definitions of contractive mappings. Trans. Am. Math. Soc. 226, 257-290 (1977)

3. Kannan, R: Some results on fixed points II. Am. Math. Mon. 76, 405-408 (1969)

4. Reich, S: Some remarks concerning contraction mappings. Can. Math. Bull. 14, 121-124 (1971)

5. Bianchini, RMT: Su un problema di S. Reich aguardante la teoría dei punti fissi. Boll. Unione Mat. Ital. 5, 103-108 (1972)

6. Chatterjea, SK: Fixed point theorems. C. R. Acad. Bulgare Sci. 25, 727-730 (1972)

7. Sehgal, VM: On fixed and periodic points for a class of mappings. J. Lond. Math. Soc. 5(2), 571-576 (1972)

8. Hardy, GE, Rogers, TD: A generalization of a fixed point theorem of Reich. Can. Math. Bull. 16, 201-206 (1973)

9. Ćirić, LB: A generalization of Banach's contraction principle. Proc. Am. Math. Soc. 45, 267-273 (1974)

10. Boyd, DW, Wong, JSW: On nonlinear contractions. Proc. Am. Math. Soc. 20, 458-464 (1969)

11. Rhoades, BE: Some theorems on weakly contractive maps. Nonlinear Anal. 47, 2683-2693 (2001)

12. Dutta, PN, Choudhury, BS: A generalization of contraction principle in metric spaces. Fixed Point Theory Appl. 2008 Article ID 406368 (2008)

13. Aydi, H, Amor, SH, Karapınar, E: Berinde-type generalized contractions on partial metric spaces. Abstr. Appl. Anal. 2013, Article ID 312479 (2013)

14. Berzig, M, Karapınar, E, Roldán-López-de-Hierro, AF: Discussion on generalized- $(\alpha \psi, \beta \varphi)$-contractive mappings via generalized altering distance function and related fixed point theorems. Abstr. Appl. Anal. 2014, Article ID 259768 (2014)

15. Dorić, D: Common fixed point for generalized $(\psi, \varphi)$-weak contractions. Appl. Math. Lett. 22, 1896-1900 (2009)

16. Karapınar, E: Fixed point theory for cyclic weak $\phi$-contraction. Appl. Math. Lett. 24(6), 822-825 (2011)

17. Aydi, H, Karapınar, E, Shatanawi, W: Coupled fixed point results for $(\psi, \varphi)$-weakly contractive condition in ordered partial metric spaces. Comput. Math. Appl. 62(12), 4449-4460 (2011)

18. Aydi, H, Postolache, M, Shatanawi, W: Coupled fixed point results for $(\psi, \phi)$-weakly contractive mappings in ordered image-metric spaces. Comput. Math. Appl. 63(1), 298-309 (2012)

19. Shatanawi, W: Some fixed point results for a generalized $\psi$-weak contraction mappings in orbitally metric spaces. Chaos Solitons Fractals 45(4), 520-526 (2012)

20. Shatanawi, W, Samet, B: On $(\psi, \phi)$-weakly contractive condition in partially ordered metric spaces. Comput. Math Appl. 62(8), 3204-3214 (2011)

21. Harjani, J, López, B, Sadarangani, K: Fixed point theorems for mixed monotone operators and applications to integral equations. Nonlinear Anal. 74(5), 1749-1760 (2011)

22. Harjani, J, Sadarangani, K: Fixed point theorems for weakly contractive mappings in partially ordered sets. Nonlinear Anal. 71, 3403-3410 (2009)

23. Aghajani, A, Radenović, S, Roshan, JR: Common fixed point results for four mappings satisfying almost generalized $(S, T)$-contractive condition in partially ordered metric spaces. Appl. Math. Comput. 218(9), 5665-5670 (2012)

24. Arandjelović, I, Kadelburg, Z, Radenović, S: Boyd-Wong-type common fixed point results in cone metric spaces. Appl. Math. Comput. 217(17), 7167-7171 (2011)

25. Roldán, A, Martínez-Moreno, J, Roldán, C, Cho, YJ: Multidimensional coincidence point results for compatible mappings in partially ordered fuzzy metric spaces. Fuzzy Sets Syst. 251, 71-82 (2014)

26. Roldán, A, Karapınar, E: Some multidimensional fixed point theorems on partially preordered $G^{*}$-metric spaces under $(\psi, \varphi)$-contractivity conditions. Fixed Point Theory Appl. 2013, Article ID 158 (2013)

27. Roldán, A, Martínez-Moreno, J, Roldán, C, Karapınar, E: Multidimensional fixed-point theorems in partially ordered complete partial metric spaces under $(\psi, \varphi)$-contractivity conditions. Abstr. Appl. Anal. 2013, Article ID 634371 (2013)

28. Karapınar, E, Roldán, A, Shahzad, N, Sintunavarat, W: Discussion of coupled and tripled coincidence point theorems for $\varphi$-contractive mappings without the mixed g-monotone property. Fixed Point Theory Appl. 2014, Article ID 92 (2014)

29. Roldán, A, Martínez-Moreno, J, Roldán, C: Tripled fixed point theorem in fuzzy metric spaces and applications. Fixed Point Theory Appl. 2013, Article ID 29 (2013)

30. Kutbi, MA, Roldán, A, Sintunavarat, W, Martínez-Moreno, J, Roldán, C: F-Closed sets and coupled fixed point theorems without the mixed monotone property. Fixed Point Theory Appl. 2013, Article ID 330 (2013)

31. Roldán-López-de-Hierro, AF, Shahzad, N: Some fixed/coincidence point theorems under $(\psi, \phi)$-contractivity conditions without an underlying metric structure. Fixed Point Theory Appl. 2014, Article ID 218 (2014)

32. Kumam, P, Roldán-López-de-Hierro, AF: On existence and uniqueness of $g$-best proximity points under $(\varphi, \theta, \alpha, g)$-contractivity conditions and consequences. Abstr. Appl. Anal. 2014, Article ID 234027 (2014)

33. Roldán, A, Martínez-Moreno, J, Roldán, C, Cho, YJ: Multidimensional fixed point theorems under $(\psi, \varphi)$-contractive conditions in partially ordered complete metric spaces. J. Comput. Appl. Math. 273, 76-87 (2015)

34. Liu, Z, Zhang, X, Ume, JS, Kang, SM: Common fixed point theorems for four mappings satisfying $\psi$-weakly contractive conditions. Fixed Point Theory Appl. 2015, Article ID 20 (2015)

35. Khan, MS, Swaleh, M, Sessa, S: Fixed point theorems by altering distances between the points. Bull. Aust. Math. Soc 30(1), 1-9 (1984)

36. Ran, ACM, Reurings, MCB: A fixed point theorem in partially ordered sets and some applications to matrix equations. Proc. Am. Math. Soc. 132, 1435-1443 (2004)

37. Nieto, JJ, Rodríguez-López, R: Contractive mapping theorem in partially ordered sets and applications to ordinary differential equations. Order 22, 223-239 (2005)

38. Samet, B, Vetro, C, Vetro, P: Fixed point theorem for $\alpha-\psi$ contractive type mappings. Nonlinear Anal. 75, 2154-2165 (2012)

39. Karapınar, E, Samet, B: Generalized $\alpha$ - $\psi$-contractive type mappings and related fixed point theorems with applications. Abstr. Appl. Anal. 2012, Article ID 793486 (2012)

40. Aydi, H, Karapınar, E, Samet, B: Fixed points for generalized $(\alpha, \psi)$-contractions on generalized metric spaces. J. Inequal. Appl. 2014, Article ID 229 (2014) 